\title{
Improved alpha-beta power reduction via combined Electrical and Ultrasonic stimulation in a Parkinsonian Cortex-Basal Ganglia-Thalamus Computational Model
}

\author{
Thomas Tarnaud, Wout Joseph, Ruben Schoeters, Luc Martens, Emmeric Tanghe
}

\begin{abstract}
Objective. To investigate computationally the interaction of combined electrical and ultrasonic modulation of isolated neurons and of the parkinsonian cortex-basal gangliathalamus loop. Approach. Continuous-wave or pulsed electrical and ultrasonic neuromodulation is applied to isolated Otsuka plateau-potential generating subthalamic nucleus (STN) and Pospischil regular, fast and low-threshold spiking cortical cells in a temporally alternating or simultaneous manner. Similar combinations of electrical/ultrasonic waveforms are applied to a parkinsonian biophysical cortex-basal ganglia-thalamus neuronal network. Ultrasound-neuron interaction is modelled respectively for isolated neurons and the neuronal network with the NICE and SONIC implementations of the bilayer sonophore underlying mechanism. Reduction in $\alpha-\beta$ spectral energy is used as a proxy to express improvement in Parkinson's disease by insonication and electrostimulation. Main results. Simultaneous electro-acoustic stimulation achieves a given level of neuronal activity at lower intensities compared to the separate stimulation modalities. Conversely, temporally alternating stimulation with $50 \mathrm{~Hz}$ electrical and ultrasound pulses is capable of eliciting $100 \mathrm{~Hz}$ STN firing rates. Furthermore, combination of ultrasound with hyperpolarizing currents can alter cortical cell relative spiking regimes. In the parkinsonian neuronal network, continuouswave and pulsed ultrasound reduce pathological oscillations by different mechanisms. High-frequency pulsed separated electrical and ultrasonic deep brain stimulation (DBS) reduce pathological $\alpha-\beta$ power by entraining STN-neurons. In contrast, continuouswave ultrasound reduces pathological oscillations by silencing the STN. Compared to the separated stimulation modalities, temporally simultaneous or alternating electro-acoustic stimulation can achieve higher reductions in $\alpha-\beta$ power for the same safety contraints on electrical/ultrasonic intensity. Significance. Focused ultrasound has the potential of becoming a non-invasive alternative of conventional DBS for the treatment of Parkinson's disease. Here, we elaborate on proposed benefits of combined electro-acoustic stimulation in terms of improved dynamic range, efficiency, spatial resolution, and neuronal selectivity.
\end{abstract}

Index Terms-Ultrasonic neuromodulation, deep brain stimulation, basal ganglia, intramembrane cavitation, computational modeling

T. Tarnaud, W. Joseph, R. Schoeters, L. Martens, and E. Tanghe are with the Department of Information Technology (INTEC-WAVES/IMEC), Ghent University/IMEC, Technologypark 126, 9052 Zwijnaarde, Belgium. E-mail: thomas.tarnaud@ugent.be

\section{INTRODUCTION}

$\mathbf{I}$ $\mathrm{N}$ the last decade, scientific interest in ultrasonic neuromodulation (UNMOD) has increased, due to its high spatial resolution (millimeter resolution in the transversal direction with a single transducer), non-invasiveness, reversibility and safety [1]-[6]. Furthermore, phased-arrays of ultrasound transducers have been used for the non-invasive ablation of brain tumours or for subthalamotomy (high intensity focused ultrasound) [7]-[10]. Consequently, similar technology could in theory be used with Low Intensity Low Frequency Ultrasound (LILFU) in order to target deep brain structures non-invasively for neuromodulation [4], [11]-[13].

For the treatment of Parkinson's disease, the subthalamic nucleus (STN) is an important target, also used in conventional (electrical) deep brain stimulation (DBS). In electrical DBS, an electrode lead is surgically implanted in the brain and connected with an implanted pulse generator via wires that run subcutaneously. Electrical pulses will then modulate the activity of the neuronal tissue surrounding the implanted lead. Conventional DBS has proven effective for the improvement of parkinsonian symptoms and motor scores. However, the surgery carries a risk of complications such as infection or haemmorhage [14]-[17]. Here, the potential application of transcranial LILFU for non-invasive deep brain stimulation has been considered before [4], [11], [13], [18]. Furthermore, recent in vivo studies in MPTP (1-methyl-4-fenyl1,2,3,6-tetrahydropyridine) lesioned parkinsonian mice have demonstrated ultrasound-induced striatal dopamine normalization [19], [20], restored locomotion activity (open field test, pole test) [19]-[22], and an increase in striatal total superoxide dismutase (T-SOD) and glutathione peroxidase (GSH-PX) (neuroprotective antioxidant enzymes) [21]. Moreover, ultrasound focused at the STN or globus pallidus (GP) downregulates the $\mathrm{Bax}$ to $\mathrm{Bcl}-2$ ratio (Bax and $\mathrm{Bcl}-2$ are proapoptotic and antiapoptotic, respectively), resulting in a reduction of cleaved-caspase 3 activity in the substantia nigra [22]. Also, in vitro, an increased dopamine release in PC12 cells upon low-intensity continuous insonication is observed in [19]. These first studies on ultrasonic neuromodulation for Parkinson's disease, indicate that transcranial LILFU could be a promising alternative to conventional electrical DBS or LDOPA (L-3,4-di-hydroxy-phenylalanine) therapy.

However, the underlying mechanism of ultrasonic neuro- 
modulation is not well understood. Several tentative biophysical mechanisms have been proposed, e.g., bilayer sonophores [23]-[25], mechanosensitivity of protein channels [26], [27], flexoelectricity [28], and thermodynamically-based models [29]. Furthermore, besides the ultrasonic harmonic pressure, other physical effects of ultrasound such as the acoustic radiation force $[30]-[32]$, acoustic streaming, and extracellular cavitation [33] can interact with the tentative biophysical underlying mechanism of UNMOD by membrane bending and stretch. Here, computational models will help in order to compare the predictions of a proposed mechanism with experimental observations. Similarly, computational models have been used to improve understanding of deep brain stimulation and Parkinson's disease, i.a., DBS-induced normalization of thalamic relay [34], [35], beta-oscillatory power [36], [37] and GPi-bursting [38], importance of antidromic activation of the hyperdirect pathway [39], [40], short-term synaptic depression and axonal failure [41], mechanisms of the generation and interaction of parkinsonian beta-oscillations [37], [42], and effects on learning and impulsivity [43], [44].

Consequently, in this study, our goal is to achieve the following two novelties. First, to investigate the capability and efficiency of ultrasonic neuromodulation to reduce betaoscillatory power, as a proxy for parkinsonian bradykinesia, in a computational model of the cortex-basal gangliathalamus neuronal (CTX-BG-TH) network. Here, we implemented a fully biophysical Hodgkin-Huxley based network of the cortex-basal ganglia-thalamus, based on earlier models of the basal ganglia [34]-[36] and of the cortex [25], [45]-[47]. In particular, a computational spiking-neuron model (as opposed to firing rate based models) of the CTX-BG-TH has been proposed in [36], with integrate-and-fire representations for the cortical cells. We included biophysical cortical Pospischilmodels [45] with cortical short-term synaptic plasiticity [46]. We opted for a fully Hodgkin-Huxley network in order to have a description of ionic channels, allowing future investigations on the mechanisms of UNMOD in the neuronal network via the interaction with mechanosensitive ion channels. For ultrasonic neuromodulation, we focus in this study on the bilayer sonophore model of intramembrane cavitation [23][25]. Out of the proposed tentative mechanisms underlying UNMOD, the bilayer sonophore model was chosen for this study because of the good correspondence between simulation results [24], [25] and in vivo experimental data [4], [5], [11], [48]-[52] for insonication of the brain (i.e., succes rate as function of intensity, frequency and pulse duration, impact of the duty-cycle on excitation versus inhibition, etc.) and because a computational multi-scale optimized framework is already developed for the simulation of this mechanism [24], [25], [53]. We investigate the relative efficiency for the reduction of parkinsonian beta-oscillations with pulsed or continuous ultrasound, compared to conventional electrical deep brain stimulation.

Second, we explore the potential benefits of applying ultrasonic neuromodulation combined with electrostimulation in tandem. Recently, in [54], ultrasonic neuromodulation was combined with transcranial magnetic stimulation (TMS) to investigate the effect of ultrasound on single-pulse motor evoked potentials and on paired-pulse TMS-metrics, such as short interval intracortical inhibition (SICI) and intracortical facilitation (ICF). In theory, UNMOD could potentially be combined with all electrostimulation technologies (TMS, tDCS, DBS, etc.), with the dual aim of improving understanding of these technologies and improving their therapeutic effects (safety, spatial resolution, etc.). For the application of non-invasive transcranial deep brain stimulation, an idea is to combine ultrasound with temporal interference deep brain stimulation (TI-DBS) [12], [55]. In TI-DBS, two high-frequency ( $f_{1}=2 \mathrm{kHz}$ and $f_{2}=f_{1}+10 \mathrm{~Hz}$ ) electrical currents are applied transcranially, resulting in maximal modulation depth by temporal interference at the targeted deep brain region, allowing non-invasive deep stimulation in mice. However, computational studies have predicted that results of TI-DBS in humans might be less favourable [56]-[58]. Besides improving the placement, waveforms, and number of the TI-DBS anodecathode pairs, another option could be to combine the benefits of TI-DBS and UNMOD by application in tandem. Here, to our best knowledge for the first time, we study the response to combined ultrasonic and electrical neuromodulation in isolated neuron models. Then, we investigate the benefits of their combined application in the neuronal CTX-BG-TH network.

\section{Methods}

\section{A. Cortex-Basal Ganglia-Thalamus Neuronal Network}

The basal ganglia are important for voluntary movement control and in the pathophysiology of Parkinson's disease. The striatum (Str) is the primary input nucleus of the basal ganglia and receives input from the motor cortex. Conversely, the internal part of the globus pallidus (GPi) is the output nucleus of the basal ganglia and sends inhibitory efferents to the thalamus (Th), resulting in a closed cortex-basal gangliathalamus loop. The basal ganglia can be divided into a direct and an indirect pathway. The former direct pathway runs from the striatum directly to the GPi. The latter indirect pathway runs from the striatum via the external part of the globus pallidus (GPe) to the GPi, as the output nucleus of the basal ganglia. There is also a hyperdirect pathway, that runs from the cortex via the subthalamic nucleus (STN) to the globus pallidus. Here, the STN is the only nucleus in the basal ganglia with excitatory efferents and is a popular target for the placement of deep brain stimulation leads for the treatment of Parkinson's disease.

A computational neuronal network model of the cortexbasal ganglia-thalamus loop was realised in the DynaSim MATLAB ${ }^{\circledR}$-toolbox for neural modeling and simulation [59] (Euler discretisation, time step $\Delta t=10 \mu$ s for the parkinsonian network, electrical DBS and continuous-wave ultrasound, $\Delta t=5 \mu \mathrm{s}$ for ultrasonic DBS, and $\Delta t=2.5 \mu \mathrm{s}$ for combined ultrasound and electrostimulation: a lower temporal discretization step is used for ultrasonic or combined electroacoustic stimulation, due to higher computational stiffness). All simulations are run for $11 \mathrm{~s}$, where initialisation-dependent network transient effects during the first second are removed. A schematic overview of the network topology is given in Fig. 1 (a). The cortical network (CTX) is based on [25], 

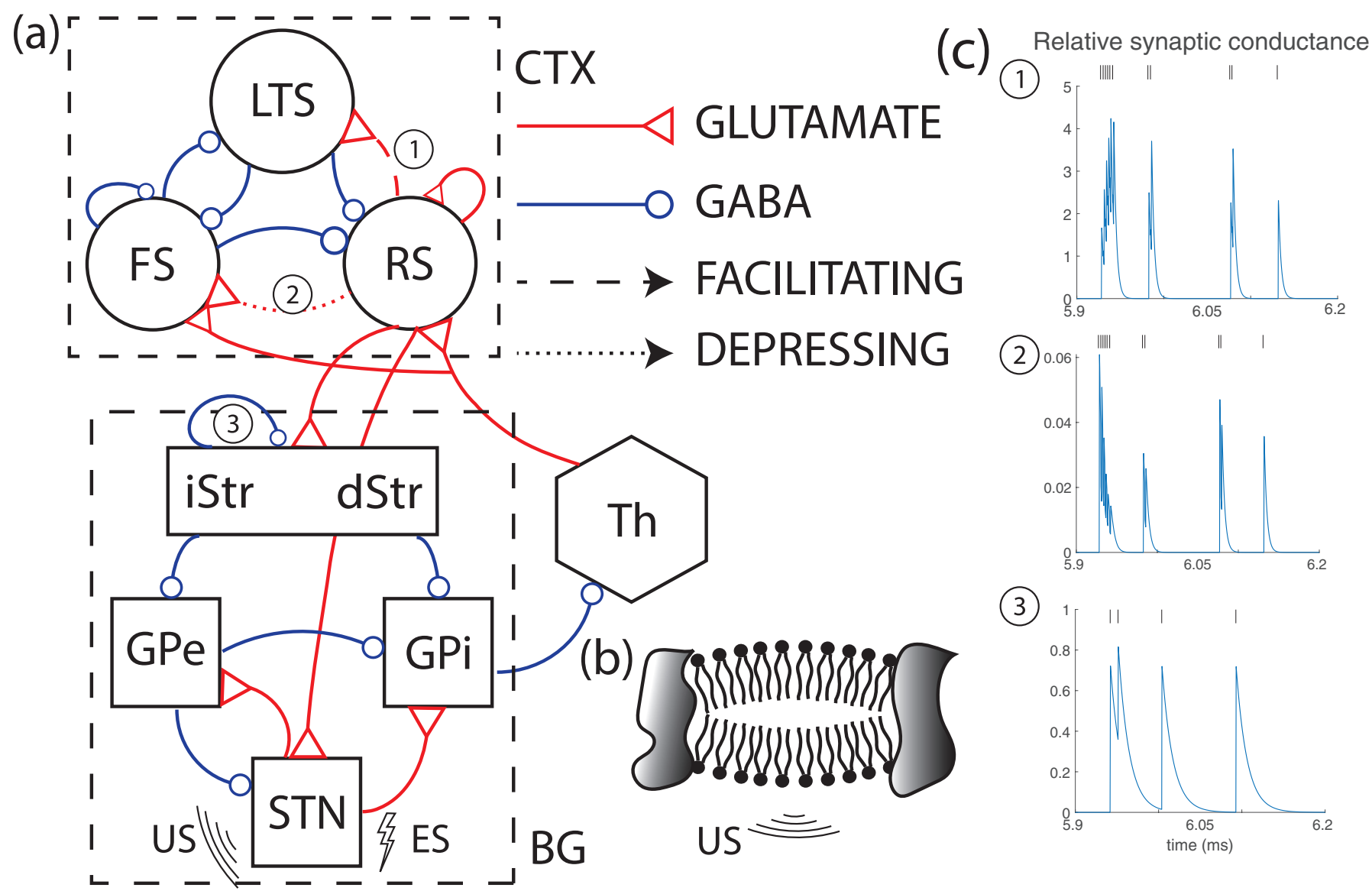

(3)

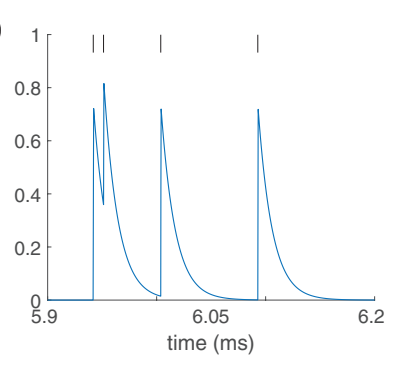

Fig. 1: (a) Schematical overview of the cortex-basal ganglia-thalamus model. GABAergic inhibitory and glutamatergic (AMPA or NMDA) excitatory synapses are depicted with circular (blue) and triangular (red) arrows, respectively. Dashed and dotted arrows are used to indicate facilitating and depressing short term synaptic plasticity. (b) Illustration of the bilayer sonophore interaction with ultrasound. The ultrasound-induced oscillations of the phospholipid bilayer is constrained by the surrounding protein islands. (c) Relative conductance $(s P)$ of a single synapse for the RS $\rightarrow$ LTS (1), RS $\rightarrow$ FS (2) and the Str $\rightarrow$ Str (3) connections. Presynaptic spikes are indicated with vertical lines. Abbreviations. CTX: cortex, RS: regular spiking, FS: fast spiking, LTS: low threshold spiking, iStr: indirect striatum, dStr: direct striatum, GPe: globus pallidus externus, GPi: globus pallidus internus, STN: subthalamic nucleus, Th: thalamus, US: ultrasonic stimulation, ES: electrical stimulation, GABA: gamma-aminobutyric acid, AMPA: alpha-amino-3-hydroxy-5-methyl-4-isoxazolepropionic acid, NMDA: N-Methyl-d-aspartic acid.

[46], [47] and consists of twenty regular spiking (RS), five fast spiking (FS), and five low-threshold spiking (LTS) cells. Cortical cell numbers are in agreement with estimates that about $30 \%$ of cortical cells are interneurons and $50 \%$ of interneurons are fast spiking [46], [60]. Basal ganglia (BG) cells included in the network are ten indirect striatal (iStr), ten direct striatal (dStr), twenty globus pallidus external (GPe), twenty globus pallidus internal (GPi) and twenty subthalamic nucleus (STN) neurons. The basal ganglia network model is based on [34]-[36], [61]. Finally, twenty thalamic (Th) cells receive afferent input from the basal ganglia output nucleus (GPi) and are connected with the regular and fast spiking cortical cells [47].

1) Network topology: Cortical network topology is based on [46], but with point neurons and a rescaled number of neurons. Synaptic connections between cortical cells are assigned by selecting source neurons at random (excluding selfconnections) with network connectivity (netcon) probabilities summarized in Table [a [46]. The number of connections between the cortex, basal ganglia and thalamus subsystems is given in Table Ib Basal ganglia model topology is deterministic: the number of synaptic connections is summarized in Table Ic [36]. Here, as in Kumaravelu et al. (2016), 50\% of globus pallidus cells chosen at random will not receive subthalamic nucleus afferents.

2) Synaptic modeling: The synaptic current $I_{\mathrm{syn}}$ is given by the product of the maximal synaptic gain $g_{\mathrm{syn}}$, a synaptic parameter $s$, a plasticity-factor $P$, and a potential term $V-E_{\mathrm{syn}}$, with $E_{\text {syn }}$ the GABAergic or glutamatergic nernst potential:

$$
I_{\text {syn }}=g_{\text {syn }} s P\left(V-E_{\text {syn }}\right)
$$

Synaptic gains $g_{\text {syn }}$ are summarized in Table ПI(a-c) for all connections in the parkinsonian network. The synaptic parameter $s$ and the plasiticity-factor $P$ are time-varying: they are calculated with the equations, described in the remainder of this subsection. 
TABLE I: Cortex-Basal Ganglia-Thalamus Network topology Source and target neurons respectively listed along rows and columns. (a) Probabilities in \%. (b-c) Number of connections. ( $\dagger$ ) $50 \%$ of the target cells do not receive afferent input.

(a) CTX netcon probabilities

(b) CTX-BG-TH connectivity

\begin{tabular}{c|cccc|ccccc} 
& RS & FS & LTS & & & Th & RS/FS & STN & (i/d)Str \\
\hline RS & 6 & 43 & 57 & & Th & 0 & 1 & 0 & 0 \\
FS & 44 & 51 & 36 & & GPi & 1 & 0 & 0 & 0 \\
LTS & 35 & 61 & 0 & & RS & 0 & 0 & 2 & 1
\end{tabular}

(c) Basal ganglia network connectivity

\begin{tabular}{c|ccccc} 
& STN & GPe & GPi & iStr & dStr \\
\hline STN & 0 & $2^{\dagger}$ & $2^{\dagger}$ & 0 & 0 \\
GPe & 2 & 2 & 2 & 0 & 0 \\
iStr & 0 & 20 & 0 & 4 & 0 \\
dStr & 0 & 0 & 20 & 0 & 3
\end{tabular}

The synaptic channel parameter $s$ is activated by presynaptic spikes. For recurrent striatal GABA-A synapses, $s$ is modeled by [36]:

$$
\frac{\mathrm{d} s}{\mathrm{~d} t}=\alpha_{\mathrm{Str}}\left[1+\tanh \frac{V_{\mathrm{pre}}}{4}\right](1-s)-\beta_{\mathrm{Str}} s .
$$

Here, $V_{\text {pre }}$ is the presynaptic potential and $\alpha_{\mathrm{Str}}=2 \mathrm{~ms}^{-1}$, $\beta_{\text {Str }}=0.0769 \mathrm{~ms}^{-1}$.

Cortical synapses are modeled as summed bi-exponentials [46]:

$$
\begin{aligned}
& s=A\left(\tau_{\mathrm{r}}, \tau_{\mathrm{d}}\right) \sum_{i=1}^{N_{\mathrm{buffer}}}\left[e^{\frac{-\left(t-t_{\mathrm{AP}}^{\mathrm{pre}}(i)-\tau_{\text {del }}\right)}{\tau_{\mathrm{d}}}} \theta\left(t-t_{\mathrm{AP}}^{\mathrm{pre}}(i)-\tau_{\mathrm{del}}\right)-\right. \\
&\left.e^{\frac{-\left(t-t_{\mathrm{AP}}^{\mathrm{pre}}(i)-\tau_{\mathrm{del}}\right)}{\tau_{\mathrm{r}}}} \theta\left(t-t_{\mathrm{AP}}^{\mathrm{pre}}(i)-\tau_{\text {del }}\right)\right]
\end{aligned}
$$

Here, $\tau_{\mathrm{r}}$ and $\tau_{\mathrm{d}}$ are the rise and decay time, respectively. $\theta$ is the Heaviside function and $A\left(\tau_{\mathrm{r}}, \tau_{\mathrm{d}}\right)=\left[\left(\tau_{\mathrm{r}} / \tau_{\mathrm{d}}\right)^{\left(-\tau_{\mathrm{r}} /\left(\tau_{\mathrm{r}}-\tau_{\mathrm{d}}\right)\right)}-\right.$ $\left.\left(\tau_{\mathrm{r}} / \tau_{\mathrm{d}}\right)^{\left(-\tau_{\mathrm{d}} /\left(\tau_{\mathrm{r}}-\tau_{\mathrm{d}}\right)\right)}\right]^{-1}$ is a normalisation factor. Synaptic delays $\tau_{\text {del }}$ are summarized in Table II (d). Presynaptic action potential times are stored in a first in, first out buffer with size $N_{\text {buffer }}=10: t_{\mathrm{AP}}^{\mathrm{pre}}(i)$ is the $i$ th presynaptic spiking time in the buffer (spikes are detected by crossing of the threshold potential $V_{\mathrm{th}}=-10 \mathrm{mV}$ ).

Similarly, an alpha-synaptic current is a special case of a biexponential synapse $\left(\tau_{\mathrm{d}} \rightarrow \tau_{\mathrm{r}}\right.$ in (3) $)$ and is given by (the normalisation factor $A$ reduces to the number of Euler $e$ ):

$s=e \sum_{i=1}^{N_{\mathrm{buffer}}}\left[\frac{\left(t-t_{\mathrm{AP}}^{\mathrm{pre}}(i)\right)}{\tau_{\mathrm{r}}} e^{\frac{-\left(t-t_{\mathrm{AP}}^{\mathrm{pre}}(i)-\tau_{\text {del }}\right)}{\tau_{\mathrm{r}}}} \theta\left(t-t_{\mathrm{AP}}^{\mathrm{pre}}(i)-\tau_{\text {del }}\right)\right]$

Glutamate synapses from pyramidal regular spiking (RS) neurons are AMPAergic (alpha-amino-3-hydroxy-5-methyl4-isoxazolepropionic acid) and have rise and decay times of $0.1 \mathrm{~ms}$ and $3 \mathrm{~ms}$, respectively. GABA-A (gammaaminobutyric acid) synapses from fast spiking (FS) interneurons have rise and decay constants of $0.5 \mathrm{~ms}$ and $8 \mathrm{~ms}$, respectively. The GABA-A synapse from low threshold spiking (LTS) interneurons has a rise and decay constant equal to $0.5 \mathrm{~ms}$ and $50 \mathrm{~ms}$ [25], [46]. A synaptic delay of $\tau_{\text {del }}=1 \mathrm{~ms}$ is introduced for cortical connections.

Short-term synaptic-plasticity is modeled as in [62], i.e., synaptic gains are multiplied with a plasticity-factor $P=F$
TABLE II: Synaptic delays and gains of the parkinsonian neuronal network.

Source and target neurons respectively listed along rows and columns. (a) Intracortical, (b) basal ganglia, and (c) cortex-basal gangliathalamus synaptical gains $\left[\mathrm{mS} / \mathrm{cm}^{2}\right]$ and (d) delays, rise and decay times [ms].

$\dagger$ Uniformly distributed around mean given in table

* Gain or time constant of AMPA and NMDA, respectively.

(a) CTX gain $\left[\mathrm{mS} / \mathrm{cm}^{2}\right]$

(b) Basal ganglia gains $\left[\mathrm{mS} / \mathrm{cm}^{2}\right]$

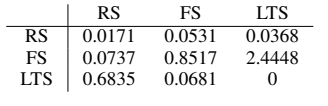

\begin{tabular}{c|ccccc} 
& STN & GPe & GPi & iStr & dStr \\
\hline STN & - & $1.089^{\dagger}, 0.00495^{\dagger *}$ & $0.3225^{\dagger}$ & - & - \\
GPe & 1.395 & 0.0375 & 0.45 & - & - \\
iStr & - & 0.225 & - & 0.025 & - \\
dStr & - & - & 0.15 & - & 0.033
\end{tabular}

(c) CTX-BG-TH gains $\left[\mathrm{mS} / \mathrm{cm}^{2}\right]$

\begin{tabular}{c|ccccc} 
& Th & RS & FS & STN & (i/d)Str \\
\hline Th & - & 0.1935 & 0.271 & - & - \\
GPi & 0.0336 & - & - & - & - \\
RS & - & - & - & $0.6041,0.0072^{*}$ & 0.0181
\end{tabular}

(d) Synaptic delays, rise and decay time constants [ms]

\begin{tabular}{c|ccc||c|ccc} 
Connection & $\tau_{\text {del }}$ & $\tau_{\mathrm{r}}$ & $\tau_{\mathrm{d}}$ & Connection & $\tau_{\text {del }}$ & $\tau_{\mathrm{r}}$ & $\tau_{\mathrm{d}}$ \\
\hline $\mathrm{GPi} \rightarrow \mathrm{Th}$ & 5 & 5 & 5 & $\mathrm{STN} \rightarrow \mathrm{GPe}$ & 2 & $0.4,2^{*}$ & $2.5,67^{*}$ \\
$\mathrm{GPe} \rightarrow \mathrm{GPe}$ & 1 & 5 & 5 & GPe $\rightarrow$ STN & 4 & 0.4 & 7.7 \\
$\mathrm{Str} \rightarrow \mathrm{GPe}$ & 5 & 5 & 5 & $\mathrm{RS} \rightarrow \mathrm{STN}$ & 5.9 & $0.5,2^{*}$ & $2.49,90^{*}$ \\
$\mathrm{STN} \rightarrow \mathrm{GPi}$ & 1.5 & 5 & 5 & $\mathrm{RS} \rightarrow \mathrm{Str}$ & 5.1 & 5 & 5 \\
$\mathrm{GPe} \rightarrow \mathrm{GPi}$ & 3 & 5 & 5 & $\mathrm{Th} \rightarrow \mathrm{RS}$ & 5.6 & 5 & 5 \\
$\mathrm{Str} \rightarrow \mathrm{GPi}$ & 4 & 5 & 5 & $\mathrm{Th} \rightarrow \mathrm{FS}$ & 5.6 & 5 & 5
\end{tabular}

(RS $\rightarrow$ LTS: facilitating connection) or $P=F \cdot D_{1} \cdot D_{2}$ (RS $\rightarrow$ FS: depressing connection), cfr. Fig. 1 $(P=1$ in connections without plasticity). Here, the dynamics of the facilitating factor $F$ is given by:

$$
\frac{\mathrm{d} F}{\mathrm{~d} t}=\frac{1-F}{\tau_{\mathrm{F}}}+f \sum_{i} \delta\left(t-t_{\mathrm{AP}}^{\mathrm{pre}}(i)\right) .
$$

The sum is over the presynaptic spike times $t_{\mathrm{AP}}^{\mathrm{pre}}(i)$ ( $\delta$ is the dirac-delta distribution). Consequently, $F$ will decay exponentially with time constant $\tau_{\mathrm{F}}$ to one in the absence of presynaptic spikes $\left(\tau_{\mathrm{F}}=200 \mathrm{~ms}\right.$ and $\tau_{\mathrm{F}}=94 \mathrm{~ms}$ for the RS $\rightarrow$ LTS and RS $\rightarrow$ FS connections, respectively). Conversely, after a presynaptic spike $F$ is incremented with $f(f=0.2$ and $f=0.5$ for $\mathrm{RS} \rightarrow$ LTS and RS $\rightarrow$ FS, respectively).

Similar dynamics holds for the depressing factor $D$, but here the effect of a presynaptic spike is multiplicative (i.e., after a presynaptic spike, $D$ is now updated by $D \rightarrow d D$ ):

$$
\frac{\mathrm{d} D}{\mathrm{~d} t}=\frac{1-D}{\tau_{\mathrm{D}}}+(d-1) D \sum_{i} \delta\left(t-t_{\mathrm{AP}}^{\mathrm{pre}}(i)\right) .
$$

The time constants are $\tau_{\mathrm{D} 1}=380 \mathrm{~ms}$ and $\tau_{\mathrm{D} 2}=9200 \mathrm{~ms}$. The multiplicative $d$-factors are equal to $d_{1}=0.46$ and $d_{2}=$ 0.975 .

Finally, all glumatergic synapses have Nernst-potential $E_{\mathrm{AMPA}}=E_{\mathrm{NMDA}}=0 \mathrm{mV}$. Glutamate-synapses are AMPAergic, except for the STN $\rightarrow$ GPe connection and the hyperdirect pathway (RS $\rightarrow$ STN) that also contain NMDA-synapses. The GABAergic interstriatal connections have $E_{\mathrm{GABA}, \mathrm{Str}}=-80 \mathrm{mV}$, while GABAergic Nernstpotentials in other nuclei are set to $E_{\mathrm{GABA}}=-85 \mathrm{mV}$. Synaptic delays, time constants and gains of the parkinsonian network are summarized in Table $\Pi$ 
3) Neuronal models: A modified Hodgkin-Huxley equation is used for the simulation of the neuronal response of the different nuclei:

$$
\frac{\mathrm{d} Q}{\mathrm{~d} t}=-\sum_{i} I_{\mathrm{m}, \mathrm{i}}-\sum_{j} I_{\mathrm{syn}, \mathrm{j}}-I_{\mathrm{app}}
$$

Here, $I_{\mathrm{m}, \mathrm{i}}$ and $I_{\mathrm{app}}$ are the neuron-type specific membrane currents and externally applied current, respectively. The externally applied current refers to afferent input that is not explicitly modeled, e.g., from the substantia nigra or the cortex. Table III lists an overview of the membrane currents present in the different models and references to their dynamics. The current $I_{\mathrm{syn}, \mathrm{j}}$ represents the received synaptic current from source population $j$.

4) Parkinson's disease and deep brain stimulation: The neuronal network of the cortex-basal ganglia-thalamus loop was made parkinsonian, by three modifications to the healthy network. First, as in Kumaravelu et al. (2016) [36], the striatal maximal M-type potassium current gain is decreased from $g_{\mathrm{M}, \mathrm{H}}=2.6 \mu \mathrm{S} / \mathrm{cm}^{2}$ in the healthy $(\mathrm{H})$ network to $g_{\mathrm{M}, \mathrm{PD}}=$ $1.5 \mu \mathrm{S} / \mathrm{cm}^{2}$ in the parkinsonian (PD) condition, caused by heightened striatal acetylcholine concentrations after dopamine depletion [36], [63], [64]. Second, an additional external current of $-2 \mu \mathrm{A} / \mathrm{cm}^{2}$ is applied to the subthalamic nucleus and globus pallidus, in order to promote parkinsonian bursting and synchronization in the network (current additional to $I_{\text {app }}$ in Table III . Third, synaptic gains in the reciprocally coupled STN - GPe feedback loop and in the long loop (hyperdirect pathway and back from thalamus to cortex) are increased to promote propagation of beta oscillatory power into the parkinsonian basal ganglia network. A similar methodology of altering synaptic gains and external applied currents to render the network parkinsonian, has been used in other computational studies of the basal ganglia [34]-[38], [40], [61], [65]

Electrical deep brain stimulation is simulated by injection of a $300 \mu \mathrm{A} / \mathrm{cm}^{2}$ current in the subthalamic nucleus cells with pulse repetition frequency between $10 \mathrm{~Hz}$ and $160 \mathrm{~Hz}$ [34][36]. Ultrasonic subthalamic nucleus deep brain stimulation is modeled with the multiScale Optimized model of Neuronal Intramembrane Cavitation (SONIC) [53], cfr. section [I-B In general in this paper, a longer pulse duration is used for ultrasound than for electrical pulses, because UNMOD is less energy efficient in comparison to electrostimulation in terms of inducing action potentials.

5) Spectral analysis and beta-oscillations: Parkinsonian bradykinesia is related with spectral density in the beta-band [69]-[72]. Here, we follow the hypothesis that cortical betaband activity will enter the dopamine-depleted basal ganglia, where it is strenghtened by the STN-GPe feedback loop [37], [65], [70], [73]. Consequently, a beta-rhythm is imposed on the pyramidal regular spiking cortical neurons, by intracellular injection of $1 \mu \mathrm{A} / \mathrm{cm}^{2}$ with normally distributed pulse repetition frequency (mean $20 \mathrm{~Hz}$, standard deviation $3 \mathrm{~Hz}$ ) and normally distributed duty cycle (mean $50 \%$, standard deviation $25 \%$ ).

Spectral analysis was performed by the multi-taper method (5 tapers, timebandwidth product equal to 3 ) in the chronux-
TABLE III: Summary of Neuron Models

Na: sodium; K: delayed-rectifier potassium; M: M-type slow noninactivating potassium; KCa: calcium dependent potassium; $\mathrm{T}$ : low-threshold T-type calcium; L: high-threshold L-type calcium; A: A-type potassium; Ca: high-threshold calcium; ahp: afterhyperpolarization; l: leak

\begin{tabular}{|c|c|c|c|}
\hline Neuron & Membrane currents & $I_{\text {app }}$ & Ref. \\
\hline RS & $\mathrm{Na}, \mathrm{K}, \mathrm{M}, \mathrm{l}$ & $0 \mu \mathrm{A} / \mathrm{cm}^{2}$ & $|\overline{25}|,|\overline{45}|$ \\
\hline $\mathrm{FS}$ & $\mathrm{Na}, \mathrm{K}, \mathrm{M}, \mathrm{l}$ & $0 \mu \mathrm{A} / \mathrm{cm}^{2}$ & $|\overline{25}|,|\overline{45}|$ \\
\hline LTS & $\mathrm{Na}, \mathrm{K}, \mathrm{M}, \mathrm{T}, \mathrm{l}$ & $0 \mu \mathrm{A} / \mathrm{cm}^{2}$ & $|25|,|45|, \mid 66$ \\
\hline STN & $\mathrm{Na}, \mathrm{K}, \mathrm{KCa}, \mathrm{T}, \mathrm{L}, \mathrm{A}, \mathrm{l}$ & $0 \mu \mathrm{A} / \mathrm{cm}^{2}$ & |36], 67$], 68$ \\
\hline GPe & $\mathrm{Na}, \mathrm{K}, \mathrm{Ca}, \mathrm{T}, \mathrm{ahp}, \mathrm{l}$ & $3 \mu \mathrm{A} / \mathrm{cm}^{2}$ & $\mid 36$ \\
\hline GPi & $\mathrm{Na}, \mathrm{K}, \mathrm{Ca}, \mathrm{T}$, ahp, $\mathrm{l}$ & $3 \mu \mathrm{A} / \mathrm{cm}^{2}$ & $|\overline{36}|$ \\
\hline Str & $\mathrm{Na}, \mathrm{K}, \mathrm{M}, \mathrm{l}$ & $0 \mu \mathrm{A} / \mathrm{cm}^{2}$ & $|36|,|64|$ \\
\hline Th & $\mathrm{Na}, \mathrm{K}, \mathrm{T}, \mathrm{l}$ & $1.2 \mu \mathrm{A} / \mathrm{cm}^{2}$ & $\mid 36$ \\
\hline
\end{tabular}

Matlab toolbox (chronux.org, [74]). As in [36], spectograms are calculated with a sliding window of $1 \mathrm{~s}$ with step size $0.1 \mathrm{~s}$ and the alpha-beta energy is the integrated spectral density over the alpha-beta band $(7 \mathrm{~Hz}-35 \mathrm{~Hz})$.

\section{B. Ultrasonic neuromodulation}

Computational modeling of ultrasonic neuromodulation is based on the tentative bilayer sonophore (BLS) underlying mechanism (cfr. Fig. 11(b)) [23]. Here, the ultrasonic pressure wave is assumed to induce a sinusoidal oscillation of the deflection of both lipid bilayer leaflets. Consequently, the membrane capacitance fluctuates in phase with the ultrasonic pressure wave, resulting in capacitive displacement currents. In the Neuronal Intramembrane Cavitation Excitation (NICE) model, these capacitive currents will result in membrane charge build-up, causing neuronal excitation [24], [25]. In this study, we adopt the NICE-framework for simulation of ultrasonic neuromodulation in isolated point neurons, as also used in previous studies [18], [23]-[25], [53], [75], [76].

The computational network model contains timescales across six orders of magnitude: a microsecond timescale of the ultrasound-neuron coupling, millisecond timescale of action potentials and membrane gating, and a second timescale of spike-frequency adaptation and network plasticity effects. Consequently, multi-scale optimization is required in order to prevent exorbitant simulation times. First, in the NICEimplementation [24], computational stiffness is decreased by the introduction of an update time step (here, $T_{\mathrm{up}}=50 \mu \mathrm{s}$ ) that decouples the mechanical (Rayleigh-Plesset) and electrodynamical (Hodgkin-Huxley) problems. Second, a multiScale Optimized model of Intramembrane Cavitation (SONIC) was proposed by [53], achieving significant further reductions of computational stiffness and simulation time. In the SONICmodel, effective voltage and rate parameters are pretabulated, the modified Hodgkin-Huxley equation is charge recasted and a Lennard-Jones fit is applied to the intramolecular pressure. The SONIC pretabulation step is performed by the parallelized solution of the Rayleigh-Plesset equation for sampled values of the sonophore radius, membrane charge, ultrasonic frequency and intensity. Consequently, the introduction of the SONICtables effectively removes the smallest microsecond timescale from the runtime, resulting in a three-order of magnitude reduction in simulation time, while maintaining qualitatively 


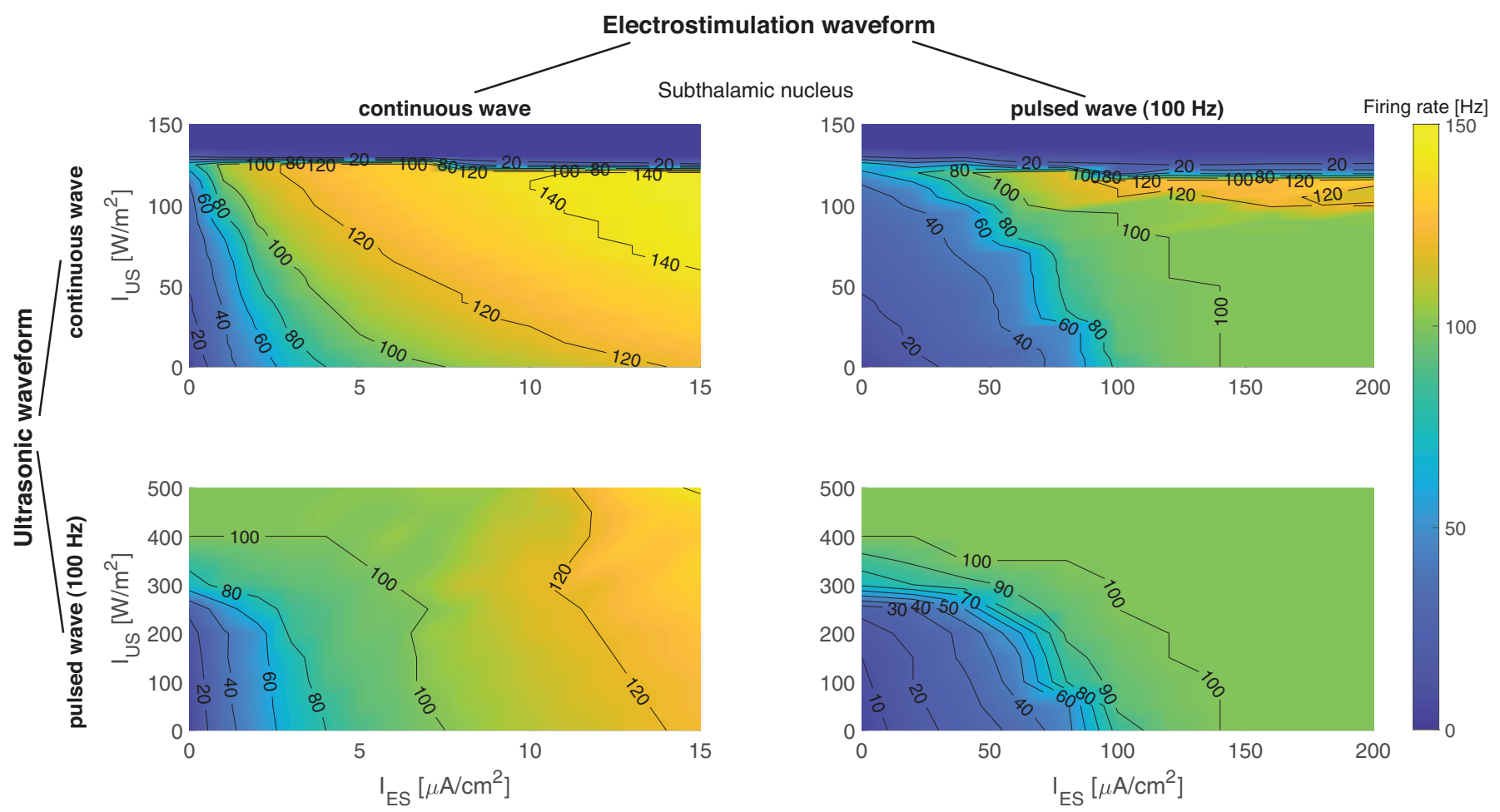

Fig. 2: Combined ultrasonic and electrical neuromodulation firing rate contour plots. Subthalamic nucleus response to simultaneous electrical and ultrasonic continuous wave or pulsed $\left(\mathrm{PRF}_{\mathrm{US}}=\mathrm{PRF}_{\mathrm{ES}}=100 \mathrm{~Hz}, f_{\mathrm{US}}=700 \mathrm{kHz}\right)$ stimulation. Electrical and ultrasonic pulses are in phase (pulse durations $\tau_{\mathrm{p}, \mathrm{ES}}=100 \mu \mathrm{s}, \tau_{\mathrm{p}, \mathrm{US}}=500 \mu \mathrm{s}$ ).

accurate results [53]. Consequently, the multi-scale optimized SONIC-model is used for all neuronal network simulations. For a description of the NICE and SONIC model, we refer to Plaksin et al. $(2014,2016)$ [24], [25] and Lemaire et al. (2019) [53], respectively.

\section{RESULTS}

\section{A. Interaction of ultrasonic and electrical neuromodulation in isolated neuron models}

In this section, we investigate the potential benefits of simultaneous electrical stimulation and insonication in isolated subthalamic nucleus point neuron models. Electro-ultrasonic stimulation in isolated cortical cells is presented in the supplementary information.

In Fig. 2, the interaction of ultrasound with electrostimulation in the plateau-potential generating subthalamic nucleus [67], [68] is shown, by plotting the firing rate contours as function of the injected electrical current and ultrasonic intensity. A non-linear interaction between continuous-wave ultrasound and continuous (direct current) electrostimulation is observed for the STN (Fig. 2(top,left)). I.e., by combining ultrasound with electrostimulation, it is possible to maintain a given level of neuronal response (e.g., $100 \mathrm{~Hz}$ spiking) at a lower ultrasonic intensity and electrical current, compared to separately applied ultrasonic neuromodulation or electrostimulation. A similar interaction between ultrasound and electrical currents is also observed for cortical cells (cfr. Supplementary Information). However, in contrast with the cortical cells, here a silenced plateau is generated for $I_{\mathrm{US}} \geq 130 \frac{\mathrm{W}}{\mathrm{m}^{2}}$ by the
STN, due to low-threshold T-type and high-threshold L-type calcium currents. This result is in agreement with our previous computational study on ultrasonic subthalamic nucleus stimulation [18]. Furthermore, combining pulsed ultrasound $(\mathrm{PRF}=100 \mathrm{~Hz})$ with DC electrical (Fig. 2(bottom,left) currents, or vice versa (Fig. 2) top,right)), can achieve $100 \mathrm{~Hz}$ pulse-locked spiking at lower pulsed intensities/currents compared to electrical or ultrasonic stimulation in separation. However, increasing the DC-current or DC-intensity, will elicit neuronal spiking at rates higher than the pulse repetition frequency. In contrast, reliable pulse-locked spiking is achieved for simultaneous and in-phase pulsed electrical and ultrasonic neurostimulation (Fig. 2(right,bottom)) at PRF $=100 \mathrm{~Hz}$. Here, by simultaneous application of ultrasound with electrical current, $100 \mathrm{~Hz}$ spiking can be elicited with lower intensities or current injections.

In Fig. 3(lower), firing rate contours of the subthalamic nucleus response to pulsed $\mathrm{PRF}=100 \mathrm{~Hz}$ alternating electrical and ultrasonic stimulation are shown. For simultaneous application of alternating electrical depolarizing pulses $\left(\mathrm{PRF}_{\mathrm{ES}}=50 \mathrm{~Hz}\right)$ of sufficient amplitude to induce pulselocked $50 \mathrm{~Hz}$ spiking and ultrasonic insonication with amplitudes at about $500 \mathrm{~W} / \mathrm{m}^{2}\left(\mathrm{PRF}_{\mathrm{US}}=50 \mathrm{~Hz}\right)$, neuronal spiking at $100 \mathrm{~Hz}$ is obtained. In Fig. 3 the different stimulation modalities (ultrasound and electrical currents) are separated in time (alternating pulses), in contrast with Fig. 2, where the concurrent electrical current and ultrasonic pressure wave stimulation modalities interact simultaneously. This separation of electrical and ultrasonic pulses is interesting from 

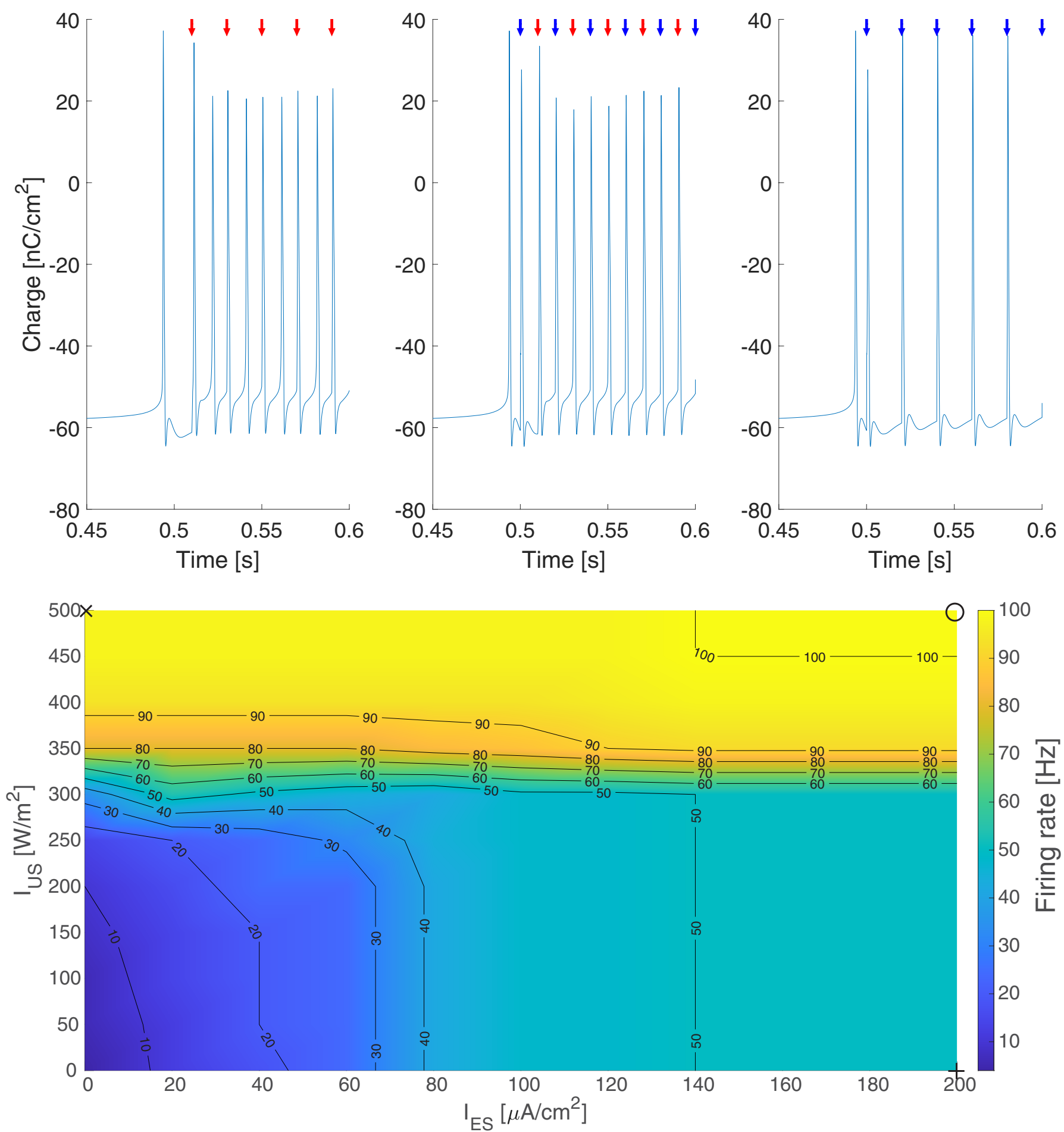

Fig. 3: Subthalamic nucleus response to combined electrical and ultrasonic pulsed out-phase stimulation (pulse repetition frequency $\mathrm{PRF}_{\mathrm{US}}=\mathrm{PRF}_{\mathrm{ES}}=50 \mathrm{~Hz}$, pulse duration $\tau_{\mathrm{p}, \mathrm{ES}}=100 \mu \mathrm{s}, \tau_{\mathrm{p}, \mathrm{US}}=500 \mu \mathrm{s}, f_{\mathrm{US}}=700 \mathrm{kHz}$ ). (Upper) Membrane charge traces. Arrows indicate the timing of ultrasonic (red) or electrical (blue) pulses. (Lower) Combined ultrasonic and electrical neuromodulation firing rate contours. Markers indicate the waveform parameters for the corresponding membrane charge traces. 

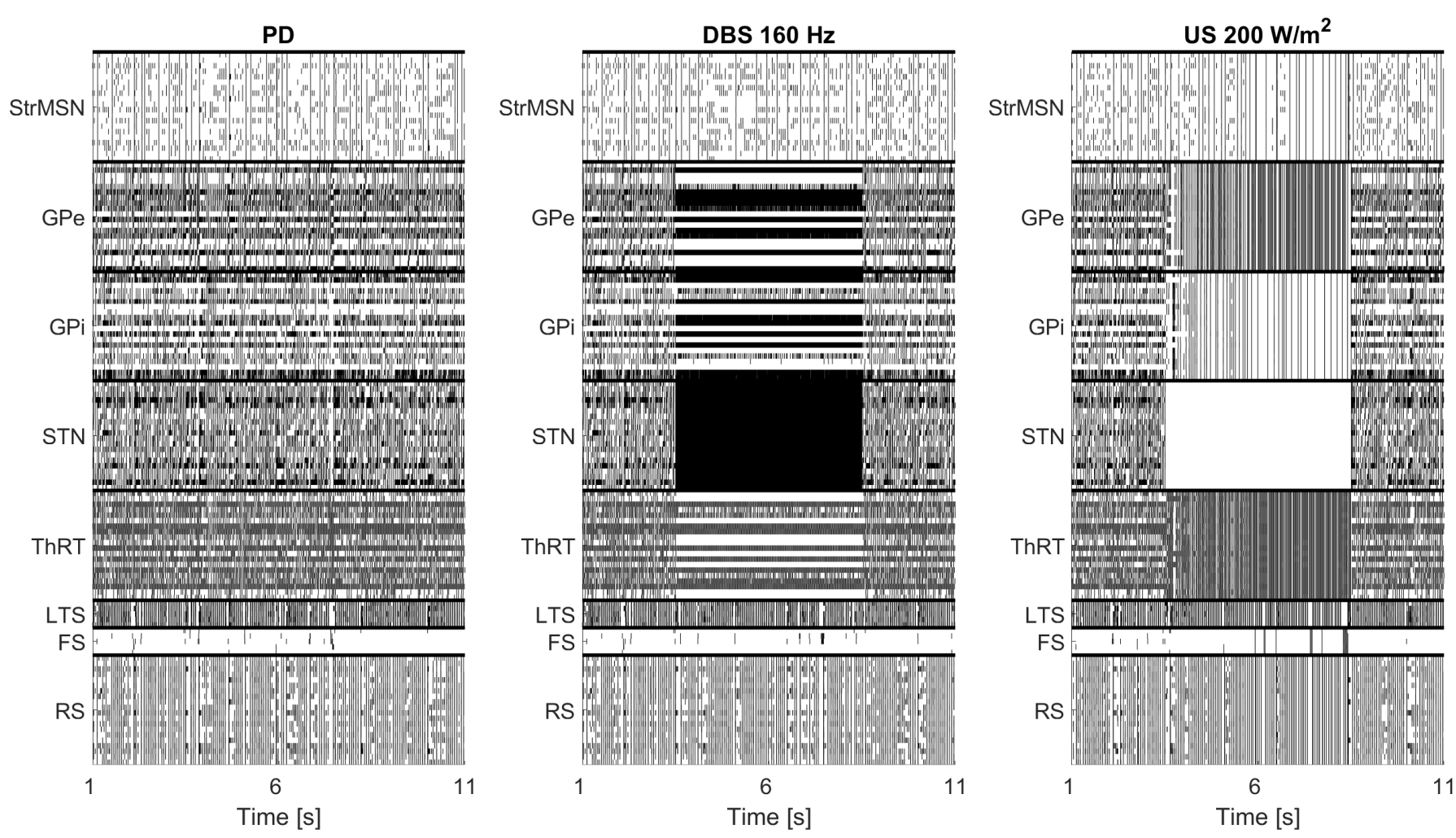

Fig. 4: Action potential rastergram (vertical lines mark the timing of neuronal spikes) for the parkinsonian (PD) cortex-basal ganglia-thalamus network without neuromodulation (left), with electrical deep brain stimulation (DBS: $\mathrm{PRF}_{\mathrm{ES}}=160 \mathrm{~Hz}$, $\tau_{\mathrm{p}, \mathrm{ES}}=300 \mu \mathrm{s}$, on time at $3.5 \mathrm{~s}$, off time at $8.5 \mathrm{~s}$; middle) and for continuous ultrasonic neuromodulation (US: $200 \mathrm{~W} / \mathrm{m}^{2}$, $f_{\mathrm{US}}=700 \mathrm{kHz}$, on time at $3.5 \mathrm{~s}$, off time at $8.5 \mathrm{~s}$; right). Abbreviations. StrMSN: striatal medium spiny neuron, GPe: globus pallidus external, GPi: globus pallidus internal, STN: subthalamic nucleus, ThRT: thalamic rubin-terman neuron, LTS: low threshold spiking cortical cell, FS: fast spiking cortical cell, RS: regular spiking cortical cell.

the perspective of safety, under the assumption that possible electrical/ultrasonic damage mechanisms are separated as well, and spatial resolution (e.g., with similar ideas to intersectional short pulse stimulation [77]). From Fig. 3 it can also be observed that pulsed ultrasound alone at $\mathrm{PRF}_{\mathrm{US}}=50 \mathrm{~Hz}$ is not capable of reliable entrainment of neuronal spiking at $50 \mathrm{~Hz}$. This observation agrees with our previous results [18], that pulse-locking for the subthalamic nucleus to ultrasound only occurs for $\mathrm{PRF}_{\mathrm{US}}>\mathrm{PRF}_{\min }>90 \mathrm{~Hz}$.

\section{B. Electrical and ultrasonic deep brain stimulation in the cortex-basal ganglia-thalamus computational network}

1) Ultrasonic and electrical stimulation separately: First, we compare the effects of ultrasonic and electrical subthalamic nucleus deep brain stimulation on the cortex-basal gangliathalamus network, when they are applied separately. Example rastergrams of the parkinsonian network in the absence of neurostimulation and in the presence of electrical $160 \mathrm{~Hz}$ and ultrasonic deep brain stimulation are shown in Fig. 4 . In Fig. 5 . the alpha-beta spectral energy $(\alpha-\beta \mathrm{SE})$, mean and standard deviation of the firing rate ( $\mu \mathrm{FR}$ and $\sigma \mathrm{FR}$, respectively) are plotted versus the pulse repetition frequency in pulsed stimulation (Fig. 5(left)) and versus the ultrasonic intensity $I_{\mathrm{US}}$ for continuous-wave ultrasonic stimulation (Fig. 5 (right)).
In the parkinsonian network, cortically imposed synchrony and beta-oscillations (e.g., see the RS trace in the PD rastergram in Fig. 4(left)) result in increased oscillations in the basal ganglia nuclei (cfr. Fig. 4(left), the parkinsonian spectrograms and the power spectral density plots in Fig. S2 in the supplementary information). In Fig. 4 electrical and ultrasonic subthalamic nucleus deep brain stimulation are applied between $t_{\text {start }}=3.5 \mathrm{~s}$ and $t_{\text {end }}=8.5 \mathrm{~s}$. Electrical pulsed $160 \mathrm{~Hz}$ deep brain stimulation entrains the subthalamic nucleus neurons at a firing rate equal to the pulse repetition frequency, with negligible variation between different STN-neuron traces (Fig. 4(middle): STN-trace). Consequently, globus pallidus neurons (GPi and GPe) that receive STN-afferents manifest tonic spiking, while globus pallidus neurons that do not receive these afferents are silenced via the GPe $\rightarrow$ GPe lateral inhibitory connections and the GPe $\rightarrow$ GPi-pathway (Fig. 4(middle): GPi and GPe). Furthermore, thalamic cells receive topologically-structured GPi GABAergic input. Therefore, the ThRT-rastergram is an inversion of the GPi-rastergram (e.g., Fig. 4(middle): ThRTtrace). The observations from the example rastergrams are confirmed and placed in context by the dependency of firing rates on pulse repetition frequency in Fig. 5(c,e): subthalamic nucleus and globus pallidus mean firing rate increases with electrical DBS pulse repetition frequency (Fig. 5(c)). While 


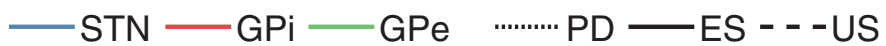

\section{Pulsed ES or US}

(a)
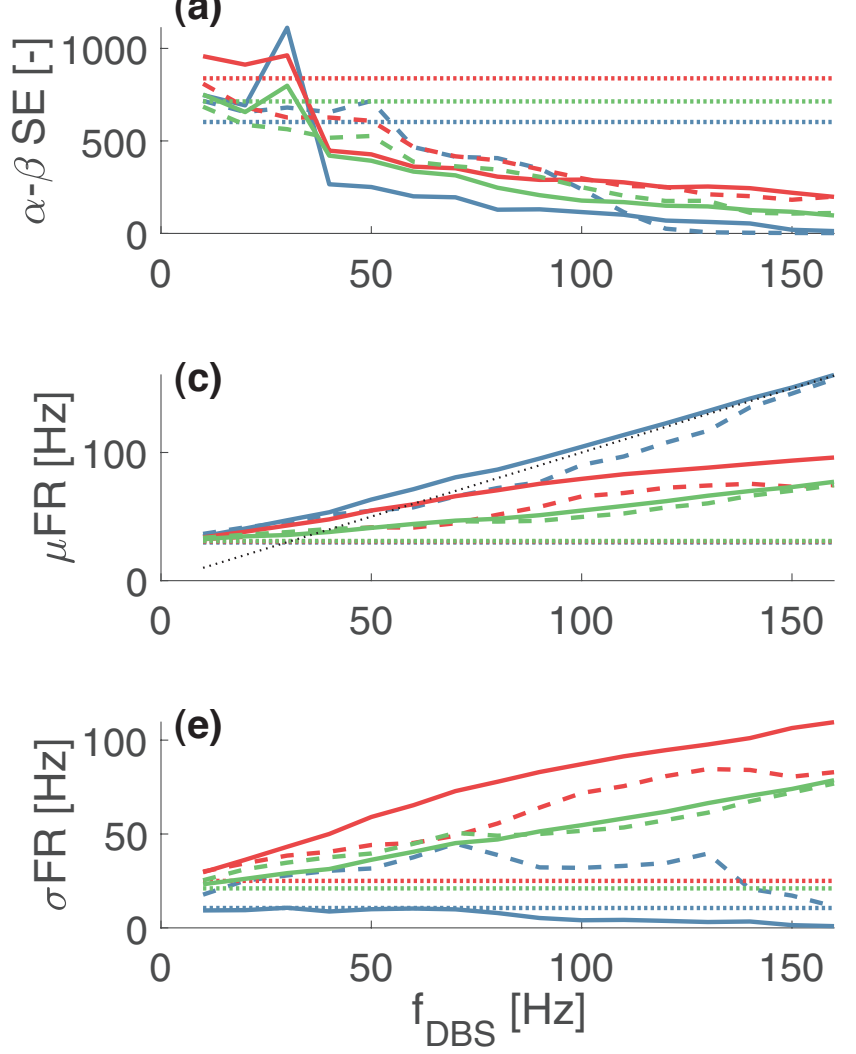

\section{Continuous US}
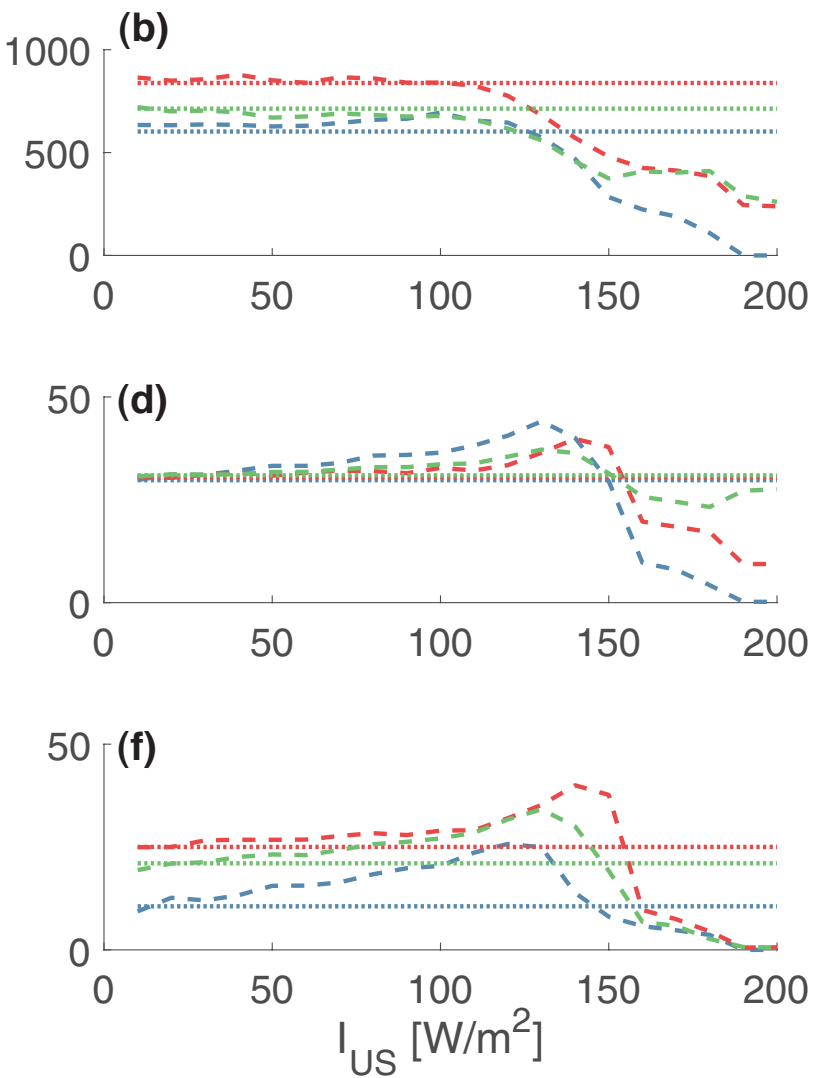

Fig. 5: Electrical and ultrasonic $\left(f_{\mathrm{US}}=700 \mathrm{kHz}\right)$ pulsed and continuous subthalamic nucleus deep brain stimulation: ES and UNMOD are applied separately. The parkinsonian network without neuromodulation is represented by dotted lines. (a) Pulsed electrostimulation $\left(\tau_{\mathrm{p}, \mathrm{ES}}=300 \mu \mathrm{s}\right)$ (full lines) or pulsed ultrasound $\left(\tau_{\mathrm{p}, \mathrm{US}}=500 \mu \mathrm{s}, I_{\mathrm{US}}=500 \mathrm{~W} / \mathrm{m}^{2}\right)$ (dashed lines) frequency-dependent effect on alpha-beta spectral energy ( $\alpha-\beta$ SE). (b) Continuous ultrasound (dashed) intensity-dependent impact on $\alpha-\beta$ SE. Mean (c-d) $(\mu \mathrm{FR})$ and standard deviation (e-f) $\sigma \mathrm{FR}$ of the firing rate versus the pulse repetition frequency in pulsed stimulation $(\mathrm{c}, \mathrm{e})$ and versus the intensity in continuous ultrasound $(\mathrm{d}, \mathrm{f})$.

STN firing rate eventually saturates to the PRF, resulting in a decrease of the variability in firing between STN-neurons with PRF (Fig. 5(c,e)), the $\sigma \mathrm{FR}$ of globus pallidus neurons increases with PRF (Fig. 5(e)). The alpha-beta spectral energy in the basal ganglia nuclei decreases with pulse repetition frequency (Fig. 5(a)), while a local worsening of $\alpha-\beta$ oscillatory power is observed around $\mathrm{PRF}=30 \mathrm{~Hz}$. The effect of $160 \mathrm{~Hz}$ electrical DBS on the power spectral density and the GPi-spectogram is shown in Fig. S2 in the supplementary information.

The results for ultrasonic pulsed deep brain stimulation are similar to electrical pulsed DBS (Fig. 5(left), dashed). Alphabeta spectral energy desirably reduces with pulse repetition frequency: here, no local worsening at low pulse repetition frequencies is observed for the GPi and GPe spectral energies, while the increase in STN beta-oscillations at low $\mathrm{PRF}$ is less pronounced. For the used ultrasonic intensity $\left(I_{\mathrm{US}}=500 \mathrm{~W} / \mathrm{m}^{2}\right)$, pulse-locking to the repetition frequency is observed at higher pulse repetition frequencies $f_{\mathrm{DBS}}$ (cfr. Fig. 5(c), blue-dashed versus black-dotted line), similar to pulsed electrostimulation. E.g., at $\mathrm{PRF}=160 \mathrm{~Hz}$, only one STN-neuron is not entrained at $160 \pm 1 \mathrm{~Hz}\left(f_{\mathrm{US}}=700 \mathrm{kHz}\right)$. In the supplementary information, we illustrate that $\alpha-\beta$ spectral energy is also already improved, without complete STN pulse-locking to the ultrasonic or electrical stimulus (Fig. S3).

Our model results predict that continuous-wave ultrasound acts by silencing subthalamic nucleus neurons to a plateau (Fig. 4(right, STN trace), Fig. 5(d)(blue dashed)). The lack of STN activity results in highly synchronized GPi and GPe activity in our network model (Fig. 4(right), GPe and GPi trace and $\sigma \mathrm{FR}$ drops to zero at higher ultrasonic pressures in Fig. 5(f)). However, alpha-beta spectral energy and firing rates are still reduced in the STN, GPi and GPe (Fig. 5 b) and Fig. 5(d), respectively). Instead, globus pallidus external oscillatory power is increased outside the alpha and beta bands, around $40 \mathrm{~Hz}$ and $78 \mathrm{~Hz}$ (cfr. Fig. S2 in the supplementary information).

2) Ultrasonic and electrical stimulation in tandem:

The application of simultaneous electrical and ultrasonic neu- 
romodulation for the suppression of $\alpha-\beta$ spectral energy in the parkinsonian network is presented in Fig. 6 and Fig. 7 for maximally out-of-phase and in-phase pulsed waveforms, respectively. From Fig. 6, we observe that STN, GPi and GPe $\alpha-\beta$-oscillations decrease with the temporally alternating electro-acoustic stimulation frequency $f_{\mathrm{EUS}}$, i.e., the pulse repetition frequency of the outphase electrical or ultrasonic pulse trains. In line with separated pulsed ES and US (Fig. 5(c),(e)), mean firing rates increase with frequency (Fig. 6(b)). Here, the firing rate variability decreases and increases with pulse repetition frequency, for the subthalamic nucleus and the globus pallidus, respectively (Fig. 6(c)). The trends for the spectral energy, mean and standard deviation of the firing rate for alternating electrical and ultrasonic pulses with $f_{\mathrm{EUS}}$ are in line with these for ultrasound or electrostimulation applied separately, but with twice the PRF (i.e., $f_{\mathrm{DBS}}=2 f_{\mathrm{EUS}}$, indicated with the upper $x$-axis in Fig. 6(a-c)).

Next, in Fig. 7phase-locked electro-ultrasonic stimulation is applied to the subthalamic nucleus neurons in the network with waveform parameters corresponding to Fig. 2(bottom right) (i.e., $\mathrm{PRF}_{\mathrm{ES}}=\mathrm{PRF}_{\mathrm{US}}=100 \mathrm{~Hz}, f_{\mathrm{US}}=700 \mathrm{kHz}, \tau_{\mathrm{p}, \mathrm{ES}}=$ $100 \mu \mathrm{s}$ and $\left.\tau_{\mathrm{p}, \mathrm{US}}=500 \mu \mathrm{s}\right)$. Here, it is interesting to observe the discrepancy in the firing rate contours of the subthalamic nucleus (cfr. Fig. 2 bottom right) and Fig. 7 (b)). In particular, while in the isolated STN $100 \mathrm{~Hz}$ firing was observed in a significant area of the explored parameter space (e.g., for $I_{\mathrm{ES}} \geq$ $150 \mu \mathrm{A} / \mathrm{cm}^{2}$, Fig. 2), similar pulse-locked firing $(100 \mathrm{~Hz} \pm$ $1 \%)$ is only reached at two simulation points $\left(\left(I_{\mathrm{ES}}, I_{\mathrm{US}}\right)=\right.$ $\left.\left(180 \mu \mathrm{A} / \mathrm{cm}^{2}, 500 \mathrm{~W} / \mathrm{m}^{2}\right),\left(200 \mu \mathrm{A} / \mathrm{cm}^{2}, 500 \mathrm{~W} / \mathrm{m}^{2}\right)\right)$ for the mean firing rate of STN-neurons that receive synaptic afferents. Furthermore, the variation in firing rate $\sigma \mathrm{FR}$ increases with electrical and ultrasonic strength in the parameter regions where pulse-locked mean firing is not achieved (Fig. 7(c)), indicating that the GABAergic and glutamatergic synaptic currents in the network model result in a reduction in reliable spiking and pulse-locking, compared to isolated STN-neurons. The GPi (Fig. 7(e,f)) and GPe (Fig. 7(h,i)) mean and standard deviation on the firing rate increase with the interacting electrical and ultrasonic intensity. Finally, we can observe from Fig. 7 $(\mathrm{a}, \mathrm{d}, \mathrm{g})$ that the parkinsonian $\alpha-\beta$ oscillations are reduced faster by combining electrical and ultrasonic stimulation (i.e., spectral energy contours are not horizontal or vertical).

\section{DISCUSSION}

\section{A. Combined UNMOD and electrostimulation}

First, interaction of simultaneous (Fig. 2) and nonsimultaneous (Fig. 3) ultrasound and electrical currents was investigated in isolated neuron models. We observe that nonlinear interaction of UNMOD with electrostimulation can achieve a given level of neuronal response (firing rate contour), at lower ultrasonic intensity and electrical current than would be required if these stimulation modalities are used separately. These results predict an increase in firing rate dynamic range for EUS-stimulation, or conversely an improvement of safety, with respect to damage mechanisms that are separated for ultrasound and electrostimulation. For example, the probability of damage by ultrasound-induced inertial cavitation and via electrochemical effects is likely not influenced by the presence of electrical current and ultrasound, respectively. Furthermore, the neuronal network simulations predict that the ultrasonic intensity required to reduce the alpha-beta spectral energy is relatively low, e.g., compared to insonication thresholds for cortical cells. This can be explained as in [18], by the observation that the subthalamic nucleus cells are spontaneously active, resulting in lower ultrasonic intensities necessary for neuromodulation. Indeed, the maximal spatial peak pulse averaged (sppa) intensity for subthalamic nucleus insonication used in this study is only $I_{\text {sppa }} \leq 0.05 \mathrm{~W} / \mathrm{cm}^{2}$ (corresponding mechanical index $\mathrm{MI}=p_{\mathrm{US}}[\mathrm{MPa}] / \sqrt{f_{\mathrm{US}}[\mathrm{MHz}]}=0.047$, $p_{\text {US }}$ is the ultrasonic pressure), which is well below the FDA safety limits for ultrasound (spatial peak pulse averaged (sppa) intensity $I_{\text {sppa,FDA }}=190 \mathrm{~W} / \mathrm{cm}^{2}$, and mechanical index $\mathrm{MI}_{\mathrm{FDA}}=1.9$ [78]). The maximal spatial peak temporal averaged (spta) intensity used in this paper for STN-stimulation is $20 \mathrm{~mW} / \mathrm{cm}^{2}$ and $4 \mathrm{~mW} / \mathrm{cm}^{2}$ for continuous and pulsed waveforms, respectively (the FDA spta-intensity guideline is $I_{\text {spta, } \mathrm{FDA}}=720 \mathrm{~mW} / \mathrm{cm}^{2}$ ).

Another potential application of the observed interaction of ultrasound with electrical currents, is the construction of stimulation modalities that leverage this interaction for improved targeting resolution (e.g., combined TI-DBS and focused ultrasound with partially or fully overlapping foci). In theory, any electrostimulation technology (transcranial magnetic stimulation (TMS), transcranial direct current stimulation (tDCS), electrical deep brain stimulation (ES-DBS), etc.) can be combined with transcranial focused ultrasound (tFUS) in order to combine the benefits and mitigate the downsides of the neurostimulation methods. E.g., tFUS has the benefit of high (millimeter size) resolution and transcranial focusing, while electrostimulation is energetically more favourable (ultrasound requires six orders of magnitude more energy than direct current injection for action potential initiation [24]).

\section{B. Deep brain stimulation in a CTX-BG-TH neuronal network}

In this study, a biophysically realistic computational model of the cortex-basal ganglia-thalamus loop was constructed, based on [25], [34]-[36], [46], [47], [61], and its interaction with ultrasound and electrostimulation was investigated. Firing rates in the healthy and parkinsonian network are in correspondence with recordings in healthy and 6-OHDA dopamine depleted rats [79]. The parkinsonian network demonstrated increased basal ganglia oscillatory beta power, that is reduced by deep brain stimulation to the STN. Here, deep brain stimulation efficiency improves with increasing pulse repetition frequency between $50 \mathrm{~Hz}$ and $160 \mathrm{~Hz}$, as observed in patients [80] and other basal ganglia models [35], [36]. Similar to electrical DBS, ultrasonic pulsed stimulation improves pathological network oscillations by driving the subthalamic nucleus spiking rate (Fig. 4/5(a,c,e)). Furthermore, interaction of temporally alternating or simultaneous ultrasonic and electrical pulses results in a higher obtained firing rate or reduction of parkinsonian oscillations for given separated limits on electrical currents and ultrasonic intensity 

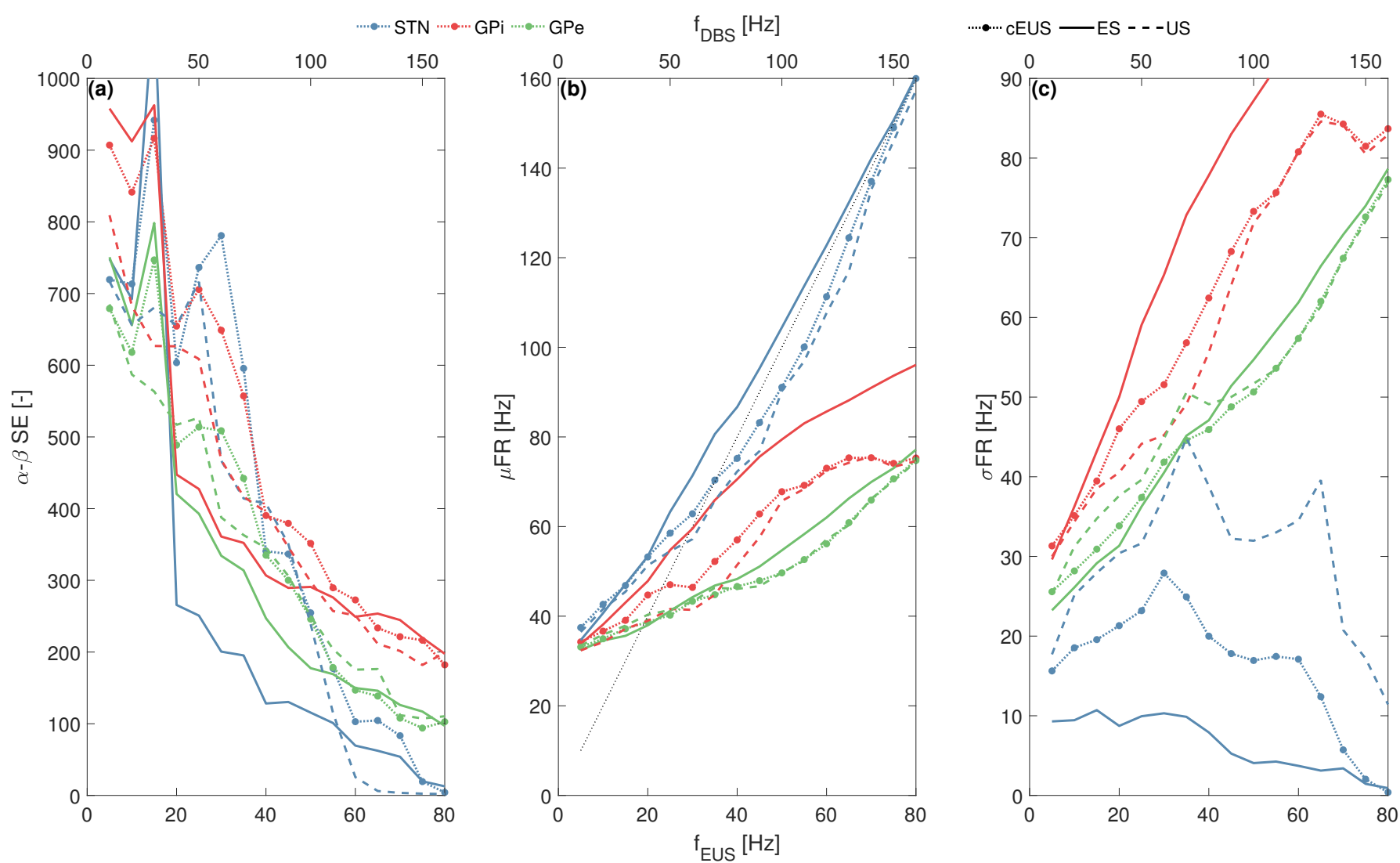

Fig. 6: Effect of temporally alternating electrostimulation $\left(\tau_{\mathrm{p}, \mathrm{ES}}=300 \mu \mathrm{s}\right)$ and ultrasonic $\left(700 \mathrm{kHz}, \tau_{\mathrm{p}, \mathrm{US}}=500 \mu \mathrm{s}\right.$, $I_{\mathrm{US}}=500 \mathrm{~W} / \mathrm{m}^{2}$ ) neuromodulation (cEUS, dotted, with marker) on alpha-beta spectral energy (a), mean firing rate $(\mu \mathrm{FR})$ (b) and standard deviation of the firing rate $(\sigma \mathrm{FR})(\mathrm{c})$, in comparison with electrostimulation (ES, full lines) and insonication (US, dashed lines) applied separately.

(Fig. 677). Here, we observe that a dichotomous response of globus pallidus neurons is obtained at therapeutic parameters (Fig. 6(c), Fig.7(f,i)), similar to Kumaravelu et al. (2016) [36]: i.e., some globus pallidus neurons exhibit tonic spiking, while others are silenced. This is not unexpected, since our basal ganglia network is based on [36], in particular the presence of two types of globus pallidus neurons, distinguished by whether or not they receive subthalamic afferents (cfr. Table I(c)).

Conversely, continuous-wave ultrasound also reduces alphabeta oscillations in our model, but by silencing the STN to a plateau-potential (Fig. 4 $4 \sqrt{5}(\mathrm{~b}, \mathrm{~d}, \mathrm{f})$ ). Here, silencing of the STN is functionally equivalent to subthalamotomy (i.e., ablation of the STN), which is an alternative treatment option for Parkinson's disease, e.g., for patients that are unsuitable for DBS-lead implantation due to access or medical reasons [81]. Computational modeling of Hahn and McIntyre (2010) also predicts a decrease in pathological globus pallidus bursting upon silencing of STN neurons [82]. However, in contrast with this result, simulations of So et al. (2012) indicate a worsening of thalamic relay error indices by removing STN local cells alone [35], speculating that lesioning of the pallidothalamic pathway is required for symptom improvement, while lesions restricted to the STN might result in dyskinesias or hemiballism. Our simulations seem to agree with Hahn and McIntyre, in the sense that beta-oscillatory power is reduced upon STN- silencing with ultrasound. However, globus pallidus synchrony is increased and strong oscillations are observed outside the alpha-beta band. Here, we hypothesize that the exact response of proxies of parkinsonian pathology to subthalamotomy might be sensitive to the values of the synaptic gains, network topology and externally applied currents. In line with this thought, So et al. observed that GPe bursting was dependent on lateral globus pallidus inhibition $\left(g_{\mathrm{GPe} \rightarrow \mathrm{GPe}}\right)[35]$. Here, the predicted correspondence of the effects of continuous and pulsed ultrasound with subthalamotomy and electrical deep brain stimulation, respectively, indicates that UNMOD could be applied for patient selection or to optimize the choice of the deep brain target.

\section{Strengths, limitations and future work}

In this paper, a fully biophysical ion-channel based neuronal network of the cortex-basal ganglia-thalamus loop has been used to quantify for the first time the applicability of ultrasonic neuromodulation for the treatment of Parkinson's disease with the alpha-beta spectral energy proxy. Furthermore, simulations in isolated neuron models and the neuronal network in this study show the benefits of simultaneous and alternating ultrasonic and electric neuromodulation.

Some important limitations and directions for future work should be taken into account. First, simulations in the cortex- 

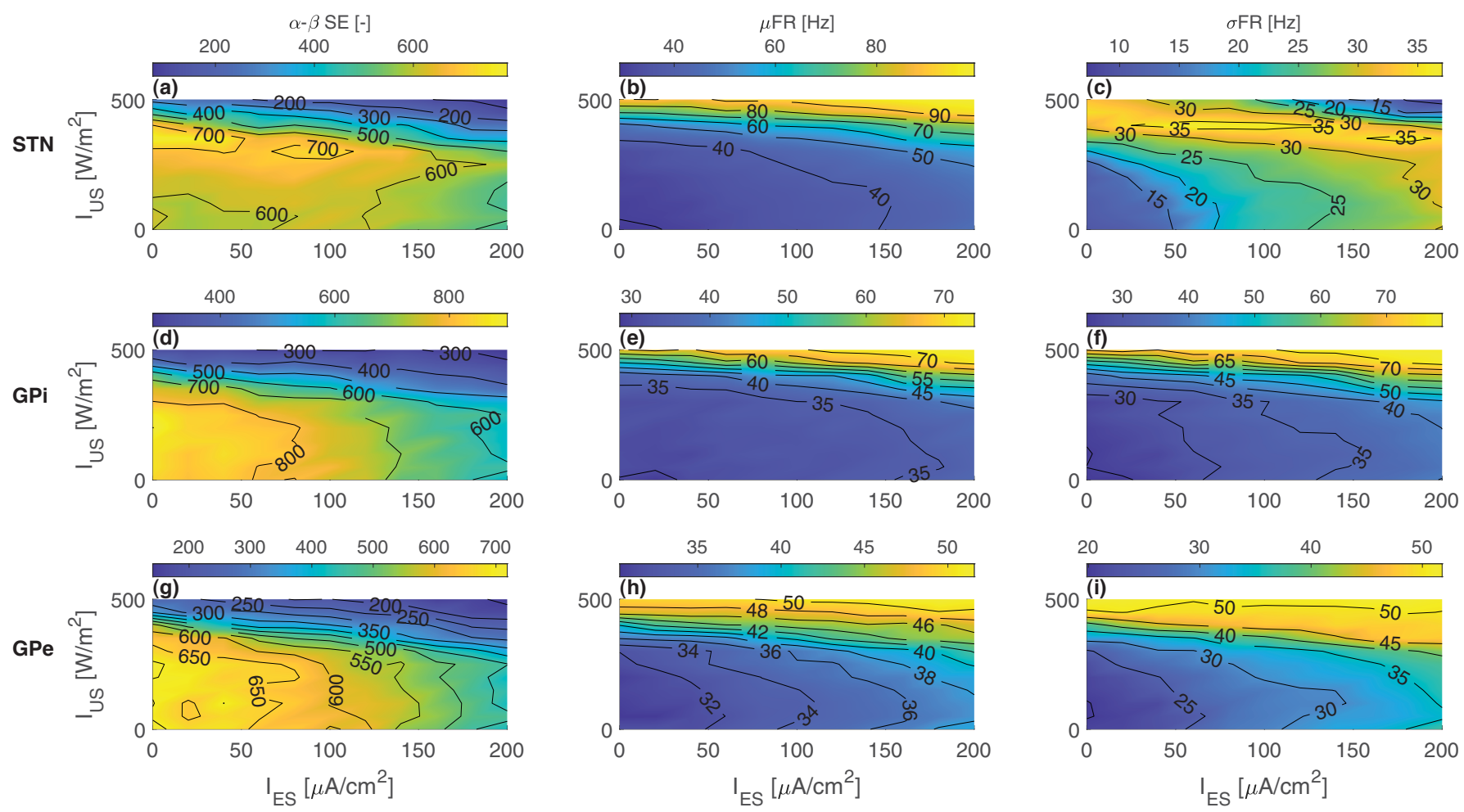

Fig. 7: Effect of phase-locked electrostimulation $\left(\tau_{\mathrm{p}, \mathrm{ES}}=100 \mu \mathrm{s}, \mathrm{PRF}_{\mathrm{ES}}=100 \mathrm{~Hz}\right)$ and ultrasonic insonication $(700 \mathrm{kHz}$, $\left.\tau_{\mathrm{p}, \mathrm{US}}=500 \mu \mathrm{s}, \mathrm{PRF}_{\mathrm{US}}=100 \mathrm{~Hz}\right)$ on alpha-beta spectral energy $(\mathrm{a}, \mathrm{d}, \mathrm{g})$, mean firing rate $(\mu \mathrm{FR})(\mathrm{b}, \mathrm{e}, \mathrm{h})$ and standard deviation of the firing rate $(\sigma \mathrm{FR})(\mathrm{c}, \mathrm{f}, \mathrm{i})$ as function of the electric current amplitude and ultrasound intensity in the subthalamic nucleus (a-c), globus pallidus interna (d-f) and globus pallidus externa (g-i).

basal ganglia-thalamus network and isolated neurons, are performed in single-compartment point neurons. Consequently, spatial effects are not taken into account and electrical deep brain stimulation is simulated by direct current injection as in earlier models [34]-[37]. Important spatial effects for electrical deep brain stimulation are antidromic propagation and cortical invasion via the hyperdirect pathway [39], [40], coactivation of fibers of passage [83] (e.g., the lenticular fasciculus (GPi $\rightarrow$ Th) passes dorsally to the subthalamic nucleus), decoupling of the somatic and axonal STN response [84], etc. Further research will also be necessary in order to determine the importance of spatial effects in ultrasonic neuromodulation (e.g., location of the excitation node and its dependence on the ultrasonic waveform). However, the construction of a multicompartmental morphologically realistic model of ultrasonic neuromodulation by intramembrane cavitation is complicated by the computational stiffness of the NICE-model, resulting in solver instabilities, low solution accuracy and/or exorbitant simulation times for neuronal models with a high number of compartments. For this reason, we designed a multi-scale optimized model SECONIC in [76] for the efficient integration of multi-compartmental UNMOD-BLS models, including fast charge oscillations and their impact on neuronal excitability. As future work, we intend to apply SECONIC to morphologically realistic models, allowing us to investigate computationally the spatial aspects of UNMOD. In this context, a recent computational study of Lemaire et al. (2021) investigated ultrasonic neuromodulation by intramembrane cavitation in multi-compartmental myelinated and unmyelinated axons with the SONIC-framework [85]. Here, spatially-extended multicompartmental neuron models could be integrated within the point neuronal network (as done in [65], for a cortical multi-compartmental cell) and coupled with finite-element and finite-difference time-domain simulations of the electric and ultrasonic field, respectively.

Second, the underlying mechanism for UNMOD is not well understood. Here, we focused on the proposed bilayer sonophore mechanism [23], [24], in which oscillating intramembrane cavities result in capacitive displacement currents and neuronal excitation. However, several other tentative mechanisms have been proposed. E.g., instead of exerting its influence by the harmonic pressure component as in the BLSmechanism, ultrasound could modulate neuronal activity via the acoustic radiation force [30], [31]. The acoustic radiation force mechanism could also be mediated via its effects on the membrane capacitance [32]. Mechanosensitivity of ion channels is another important tentative mechanism [26], [27], [86], in which the harmonic pressure component or acoustic radiation force alters the dynamics of the ion channels. Here, interaction with the bilayer sonophore model or Prieto-model of acoustic radiation force effect is possible: e.g., the NICEmodel predicts oscillating membrane tension, which could impact the mechanosensitive ion channels. Furthermore, also flexoelectricity [28], thermodynamic neuron models [29], mechanical surface and axial waves [87], [88], propagation via 
acto-myosin cytoskeletal elements [89], etc., could be important for a complete understanding of ultrasonic neuromodulation. Here, computational modeling can be helpful to improve understanding of the consequences and potential interactions of the different tentative mechanisms. In this study, predictions have been made on the interaction of bilayer sonophore mediated ultrasonic neuromodulation and electrical currents, the comparative efficiency for the reduction of beta-oscillations of UNMOD w.r.t. electrostimulation, efficacy of continuous-wave ultrasound versus pulsed ultrasound, etc. These predictions could be tested in future experimental studies. As future work, we intend to incorporate also other tentative mechanisms of ultrasound (acoustic radiation force, mechanosensitivity of ion channels...) into the models, in order to investigate the interactions between them and to gain understanding under which conditions (ultrasonic waveform, neuron type,...) a certain mechanism is expected to be dominant. Our motivation for starting our study on the interaction of ultrasound with the parkinsonian neuronal network with the BLS underlying mechanism, is based on the fact that a computational multiscale optimized framework is already developed for its simulation [24], [25], [53] and on the good correspondence between simulation results [24], [25] and in vivo experimental data [4], [5], [11], [48]-[52] for insonication of the brain (i.e., succes rate as function of intensity, frequency and pulse duration, impact of the duty cycle on excitation versus inhibition, etc.). Despite this good correspondence with experimental evidence, we would argue that this does not unequivocally prove that the bilayer sonophore model is the only dominant underlying mechanism of ultrasonic neuromodulation in the brain. It is more likely that several mechanisms will act simultaneously and that their relative importance is strongly dependent on the ultrasonic waveform, experimental conditions (in vivo or in vitro), neuron types (peripheral nerves, retina or the brain), etc. However, given the current evidence [4], [5], [11], [48]-[52], the bilayer sonophore model seems to be a good candidate to be a dominant underlying mechanism for UNMOD in vivo in the brain, while other mechanisms might be dominant in the peripheral nerves or in the retina [30], [33].

Third, in this study, electro-ultrasonic stimulation is investigated by using square-wave electrical current trains. For example, spatial resolution could be further improved by using temporally interfering waveforms in combination with ultrasound. However, because temporal interference stimulation is a recent methodology for the application of non-invasive deep brain stimulation, different research groups are still investigating the optimization of stimulus waveforms and electrode configurations [90]-[94]. Furthermore, also the underlying mechanism of temporal interference DBS is not yet understood. For example, further research is necessary on which non-linearity is able to explain why neurons can react to the beat frequency of the temporally interfering waveform (e.g., a non-linearity in the protein rate functions, in the currentmembrane voltage relation (Goldman-Hodgkin-Katz relation), etc.) and to determine which neuron types are most sensitive to a beating sinusoid [58], [95]-[97]. For these reasons, this study was not performed with temporally interfering waveforms, but with simpler rectangular pulse trains. However, we intend to investigate this topic of combining temporally interfering electrical currents with ultrasound as future work.

\section{CONClusion}

In this study, a computational biophysical (i.e., fully Hodgkin-Huxley) neuronal network of the parkinsonian cortex, basal ganglia and thalamus was constructed and coupled with ultrasound and electrostimulation. Here, the basal ganglia receive cortical input via the hyperdirect and (in)direct pathways, while the thalamus relays the output from the globus pallidus back to regular spiking pyramidal cortical cells and fast spiking interneurons. Both electrical and ultrasonic pulsed STN-stimulation is capable of reducing elevated alpha-beta pathological oscillations at higher pulse repetition frequencies. Continuous-wave ultrasound is also able of improving alphabeta power, but by the opposing mechanism of silencing the subthalamic nucleus to an elevated plateau-potential. In both the neuronal network and in isolated neuron models it was observed that simultaneous or alternating application of electro-acoustic waveforms is capable of obtaining higher firing rates and better reduction in parkinsonian oscillations for given safety limits on the electrical current and ultrasonic intensity. Furthermore, it was demonstrated in cortical cells that combining hyperpolarizing (negative) electrode currents with ultrasound can achieve altered relative cortical spiking regimes: i.e., entraining regular and fast spiking neurons to a high-frequency pulse train, while low-threshold spiking cells are quiescent.

The results presented in this study indicate that the combination of electrical and ultrasonic modalities has potential to improve safety and dynamic range, targeting resolution, recruitment selectivity, and energy efficiency. Furthermore, it is predicted that both continuous and pulsed transcranial focused ultrasound targeted to the STN is able to improve parkinsonian symptoms. Our computational model provides novel testable predictions, that can be used to verify or falsify the proposed underlying mechanisms of DBS and ultrasoundneuron interaction.

As future work, we intend to perform a sensitivity study of the efficiency of ultrasonic and electrical stimulation to variations in the network topology and synaptic gains. Here, these variations could potentially be interpreted as inter-subject variability. Furthermore, target locations other than the STN (e.g., globus pallidus or thalamus) can be investigated, as well as co-stimulation of fibres of passage.

\section{Data AVAilability STATEMEnT}

Matlab code implementing the neuronal cortex-basal ganglia-thalamus network will be made available via the modelDB database (http://modeldb.yale.edu/267195, accession nr. 267195).

\section{CONFLICT OF INTERESTS}

The authors declare that there is no conflict of interest regarding the publication of this paper. 


\section{ACKNOWLEDGMENT}

This work was carried out using the Supercomputer Infrastructure (STEVIN) at Ghent University, funded by Ghent University, the Flemish Supercomputer Center (VSC), the Hercules Foundation and the Flemish Government department EWI.

This research was funded by the FWO-project G046816N. T. Tarnaud is a PhD Fellow of the FWO-V (SB) (Research Foundation Flanders, Belgium). R. Schoeters is a PhD Fellow of the FWO-V (Research Foundation Flanders, Belgium).

\section{REFERENCES}

[1] W. J. Tyler, Y. Tufail, M. Finsterwald, M. L. Tauchmann, E. J. Olson, and C. Majestic, "Remote excitation of neuronal circuits using low-intensity, low-frequency ultrasound," PloS one, vol. 3, no. 10, p. e3511, 2008.

[2] Y. Tufail, A. Matyushov, N. Baldwin, M. L. Tauchmann, J. Georges, A. Yoshihiro, S. I. H. Tillery, and W. J. Tyler, "Transcranial pulsed ultrasound stimulates intact brain circuits," Neuron, vol. 66, no. 5, pp. 681-694, 2010.

[3] W. Legon, T. F. Sato, A. Opitz, J. Mueller, A. Barbour, A. Williams, and W. J. Tyler, "Transcranial focused ultrasound modulates the activity of primary somatosensory cortex in humans," Nature neuroscience, vol. 17, no. 2, p. 322, 2014

[4] R. L. King, J. R. Brown, W. T. Newsome, and K. B. Pauly, "Effective parameters for ultrasound-induced in vivo neurostimulation," Ultrasound in medicine \& biology, vol. 39, no. 2, pp. 312-331, 2013.

[5] H. Kim, A. Chiu, S. D. Lee, K. Fischer, and S.-S. Yoo, "Focused ultrasound-mediated non-invasive brain stimulation: examination of sonication parameters," Brain stimulation, vol. 7, no. 5, pp. 748-756, 2014.

[6] W. Legon, S. Adams, P. Bansal, P. D. Patel, L. Hobbs, L. Ai, J. K. Mueller, G. Meekins, and B. T. Gillick, "A retrospective qualitative report of symptoms and safety from transcranial focused ultrasound for neuromodulation in humans," Scientific reports, vol. 10, no. 1, pp. 1-10, 2020.

[7] M. Pernot, J.-F. Aubry, M. Tanter, J.-L. Thomas, and M. Fink, "High power transcranial beam steering for ultrasonic brain therapy," Physics in medicine and biology, vol. 48, no. 16, p. 2577, 2003.

[8] L. Marsac, D. Chauvet, B. Larrat, M. Pernot, B. Robert, M. Fink, A.L. Boch, J.-F. Aubry, and M. Tanter, "Mr-guided adaptive focusing of therapeutic ultrasound beams in the human head," Medical physics, vol. 39, no. 2, pp. 1141-1149, 2012.

[9] G. T. Clement, P. J. White, R. L. King, N. McDannold, and K. Hynynen, "A magnetic resonance imaging-compatible, large-scale array for transskull ultrasound surgery and therapy," Journal of ultrasound in medicine, vol. 24, no. 8, pp. 1117-1125, 2005.

[10] K. Hynynen, G. T. Clement, N. McDannold, N. Vykhodtseva, R. King, P. J. White, S. Vitek, and F. A. Jolesz, "500-element ultrasound phased array system for noninvasive focal surgery of the brain: A preliminary rabbit study with ex vivo human skulls," Magnetic Resonance in Medicine: An Official Journal of the International Society for Magnetic Resonance in Medicine, vol. 52, no. 1, pp. 100-107, 2004.

[11] R. L. King, J. R. Brown, and K. B. Pauly, "Localization of ultrasoundinduced in vivo neurostimulation in the mouse model," Ultrasound in medicine \& biology, vol. 40, no. 7, pp. 1512-1522, 2014.

[12] N. Grossman, D. Bono, N. Dedic, S. B. Kodandaramaiah, A. Rudenko, H.-J. Suk, A. M. Cassara, E. Neufeld, N. Kuster, L.-H. Tsai et al., "Noninvasive deep brain stimulation via temporally interfering electric fields," Cell, vol. 169, no. 6, pp. 1029-1041, 2017.

[13] M. A. Samoudi, T. Van Renterghem, and D. Botteldooren, "Computational modeling of a single-element transcranial focused ultrasound transducer for subthalamic nucleus stimulation," Journal of neural engineering, 2018.

[14] J. M. Bronstein, M. Tagliati, R. L. Alterman, A. M. Lozano, J. Volkmann, A. Stefani, F. B. Horak, M. S. Okun, K. D. Foote, P. Krack et al., "Deep brain stimulation for parkinson disease: an expert consensus and review of key issues," Archives of neurology, vol. 68, no. 2, pp. 165-165, 2011.

[15] K. Sugiyama, "Complications of deep brain stimulation," in Deep Brain Stimulation for Neurological Disorders. Springer, 2015, pp. 195-206.

[16] A. L. Benabid, S. Chabardes, J. Mitrofanis, and P. Pollak, "Deep brain stimulation of the subthalamic nucleus for the treatment of parkinson's disease," The Lancet Neurology, vol. 8, no. 1, pp. 67-81, 2009.
[17] G. Kleiner-Fisman, J. Herzog, D. N. Fisman, F. Tamma, K. E. Lyons, R. Pahwa, A. E. Lang, and G. Deuschl, "Subthalamic nucleus deep brain stimulation: summary and meta-analysis of outcomes," Movement disorders: official journal of the Movement Disorder Society, vol. 21, no. S14, pp. S290-S304, 2006.

[18] T. Tarnaud, W. Joseph, L. Martens, and E. Tanghe, "Computational modeling of ultrasonic subthalamic nucleus stimulation," IEEE Transactions on Biomedical Engineering, vol. 66, no. 4, pp. 1155-1164, 2018.

[19] T. Xu, X. Lu, D. Peng, G. Wang, C. Chen, W. Liu, W. Wu, and T. J. Mason, "Ultrasonic stimulation of the brain to enhance the release of dopamine-a potential novel treatment for parkinson's disease," Ultrasonics Sonochemistry, vol. 63, p. 104955, 2020.

[20] W. Wang, L. Li, W. Wu, W. Zhang, Y. Gao, and C. Chen, "Effects of ultrasound on behavior and dopamine content in striatum of parkinson's disease model mouse," in 2017 International Conference on Material Science, Energy and Environmental Engineering (MSEEE 2017). Atlantis Press, 2017

[21] H. Zhou, L. Niu, X. Xia, Z. Lin, X. Liu, M. Su, R. Guo, L. Meng, and H. Zheng, "Wearable ultrasound improves motor function in an mptp mouse model of parkinson's disease," IEEE Transactions on Biomedical Engineering, vol. 66, no. 11, pp. 3006-3013, 2019.

[22] H. Zhou, L. Niu, L. Meng, Z. Lin, J. Zou, X. Xia, X. Huang, W. Zhou, T. Bian, H. Zheng et al., "Noninvasive ultrasound deep brain stimulation for the treatment of parkinson's disease model mouse," Research, vol. 2019, p. 1748489, 2019.

[23] B. Krasovitski, V. Frenkel, S. Shoham, and E. Kimmel, "Intramembrane cavitation as a unifying mechanism for ultrasound-induced bioeffects," Proceedings of the National Academy of Sciences, vol. 108, no. 8, pp. 3258-3263, 2011.

[24] M. Plaksin, S. Shoham, and E. Kimmel, "Intramembrane cavitation as a predictive bio-piezoelectric mechanism for ultrasonic brain stimulation," Physical review $X$, vol. 4, no. 1, p. 011004, 2014.

[25] M. Plaksin, E. Kimmel, and S. Shoham, "Cell-type-selective effects of intramembrane cavitation as a unifying theoretical framework for ultrasonic neuromodulation," eNeuro, vol. 3, no. 3, pp. ENEURO-0136, 2016.

[26] M. L. Prieto, K. Firouzi, B. T. Khuri-Yakub, and M. Maduke, "Activation of piezo1 but not nav1. 2 channels by ultrasound at $43 \mathrm{mhz}$," Ultrasound in medicine \& biology, vol. 44, no. 6, pp. 1217-1232, 2018.

[27] J. Kubanek, J. Shi, J. Marsh, D. Chen, C. Deng, and J. Cui, "Ultrasound modulates ion channel currents," Scientific reports, vol. 6, no. 1, pp. 114, 2016.

[28] A. G. Petrov, "Flexoelectricity of model and living membranes," Biochimica et Biophysica Acta (BBA)-Biomembranes, vol. 1561, no. 1, pp. 1-25, 2002.

[29] T. Heimburg and A. D. Jackson, "On soliton propagation in biomembranes and nerves," Proceedings of the National Academy of Sciences of the United States of America, vol. 102, no. 28, pp. 9790-9795, 2005.

[30] M. D. Menz, P. Ye, K. Firouzi, A. Nikoozadeh, K. B. Pauly, P. KhuriYakub, and S. A. Baccus, "Radiation force as a physical mechanism for ultrasonic neurostimulation of the ex vivo retina," Journal of Neuroscience, vol. 39, no. 32, pp. 6251-6264, 2019.

[31] H. Chen, D. Garcia-Gonzalez, and A. Jérusalem, "Computational model of the mechanoelectrophysiological coupling in axons with application to neuromodulation," Phys. Rev. E, vol. 99, p. 032406, Mar 2019. [Online]. Available: https://link.aps.org/doi/10.1103/PhysRevE. 99.032406

[32] M. L. Prieto, Ö. Oralkan, B. T. Khuri-Yakub, and M. C. Maduke, "Dynamic response of model lipid membranes to ultrasonic radiation force," PLoS One, vol. 8, no. 10, p. e77115, 2013.

[33] C. J. Wright, S. R. Haqshenas, J. Rothwell, and N. Saffari, "Unmyelinated peripheral nerves can be stimulated in vitro using pulsed ultrasound," Ultrasound in medicine \& biology, vol. 43, no. 10, pp. 2269-2283, 2017.

[34] J. E. Rubin and D. Terman, "High frequency stimulation of the subthalamic nucleus eliminates pathological thalamic rhythmicity in a computational model," Journal of computational neuroscience, vol. 16, no. 3, pp. 211-235, 2004.

[35] R. Q. So, A. R. Kent, and W. M. Grill, "Relative contributions of local cell and passing fiber activation and silencing to changes in thalamic fidelity during deep brain stimulation and lesioning: a computational modeling study," Journal of computational neuroscience, vol. 32, no. 3, pp. 499-519, 2012.

[36] K. Kumaravelu, D. T. Brocker, and W. M. Grill, "A biophysical model of the cortex-basal ganglia-thalamus network in the 6-ohda lesioned rat model of parkinson's disease," Journal of computational neuroscience, vol. 40, no. 2 , pp. 207-229, 2016. 
[37] G. Kang and M. M. Lowery, "Interaction of oscillations, and their suppression via deep brain stimulation, in a model of the corticobasal ganglia network," IEEE Transactions on Neural Systems and Rehabilitation Engineering, vol. 21, no. 2, pp. 244-253, 2013.

[38] P. J. Hahn and C. C. McIntyre, "Modeling shifts in the rate and pattern of subthalamopallidal network activity during deep brain stimulation," Journal of computational neuroscience, vol. 28, no. 3, pp. 425-441, 2010.

[39] R. W. Anderson, A. Farokhniaee, K. Gunalan, B. Howell, and C. C. McIntyre, "Action potential initiation, propagation, and cortical invasion in the hyperdirect pathway during subthalamic deep brain stimulation," Brain stimulation, vol. 11, no. 5, pp. 1140-1150, 2018.

[40] G. Kang and M. Lowery, "Effects of antidromic and orthodromic activation of stn afferent axons during dbs in parkinson's disease: a simulation study," Frontiers in computational neuroscience, vol. 8, p. 32, 2014.

[41] R. Rosenbaum, A. Zimnik, F. Zheng, R. S. Turner, C. Alzheimer, B. Doiron, and J. E. Rubin, "Axonal and synaptic failure suppress the transfer of firing rate oscillations, synchrony and information during high frequency deep brain stimulation," Neurobiology of disease, vol. 62, pp. 86-99, 2014.

[42] A. Pavlides, S. J. Hogan, and R. Bogacz, "Computational models describing possible mechanisms for generation of excessive beta oscillations in parkinson's disease," PLoS computational biology, vol. 11, no. 12, p. e1004609, 2015.

[43] M. J. Frank, J. Samanta, A. A. Moustafa, and S. J. Sherman, "Hold your horses: impulsivity, deep brain stimulation, and medication in parkinsonism," science, vol. 318, no. 5854, pp. 1309-1312, 2007.

[44] M. J. Frank, L. C. Seeberger, and R. C. O'reilly, "By carrot or by stick: cognitive reinforcement learning in parkinsonism," Science, vol. 306, no. 5703, pp. 1940-1943, 2004.

[45] M. Pospischil, M. Toledo-Rodriguez, C. Monier, Z. Piwkowska, T. Bal, Y. Frégnac, H. Markram, and A. Destexhe, "Minimal hodgkin-huxley type models for different classes of cortical and thalamic neurons," Biological cybernetics, vol. 99, no. 4-5, pp. 427-441, 2008.

[46] D. Vierling-Claassen, J. Cardin, C. I. Moore, and S. R. Jones, "Computational modeling of distinct neocortical oscillations driven by celltype selective optogenetic drive: separable resonant circuits controlled by low-threshold spiking and fast-spiking interneurons," Frontiers in human neuroscience, vol. 4, p. 198, 2010.

[47] I. Hayut, E. E. Fanselow, B. W. Connors, and D. Golomb, "Lts and fs inhibitory interneurons, short-term synaptic plasticity, and cortical circuit dynamics," PLoS Comput Biol, vol. 7, no. 10, p. e1002248, 2011.

[48] S.-S. Yoo, A. Bystritsky, J.-H. Lee, Y. Zhang, K. Fischer, B.-K. Min, N. J. McDannold, A. Pascual-Leone, and F. A. Jolesz, "Focused ultrasound modulates region-specific brain activity," Neuroimage, vol. 56, no. 3, pp. 1267-1275, 2011.

[49] H. Kim, S. J. Taghados, K. Fischer, L.-S. Maeng, S. Park, and S.-S Yoo, "Noninvasive transcranial stimulation of rat abducens nerve by focused ultrasound," Ultrasound in medicine \& biology, vol. 38, no. 9 , pp. 1568-1575, 2012

[50] H. Kim, M. Y. Park, S. D. Lee, W. Lee, A. Chiu, and S.-S. Yoo, "Suppression of eeg visual-evoked potentials in rats via neuromodulatory focused ultrasound," Neuroreport, vol. 26, no. 4, p. 211, 2015.

[51] Y. Tufail, A. Yoshihiro, S. Pati, M. M. Li, and W. J. Tyler, "Ultrasonic neuromodulation by brain stimulation with transcranial ultrasound," nature protocols, vol. 6, no. 9, pp. 1453-1470, 2011.

[52] W. Lee, H. Kim, Y. Jung, I.-U. Song, Y. A. Chung, and S.-S. Yoo, "Image-guided transcranial focused ultrasound stimulates human primary somatosensory cortex," Scientific reports, vol. 5, no. 1, pp. 1-10, 2015.

[53] T. Lemaire, E. Neufeld, N. Kuster, and S. Micera, "Understanding ultrasound neuromodulation using a computationally efficient and interpretable model of intramembrane cavitation," Journal of Neural Engineering, 2019.

[54] W. Legon, P. Bansal, R. Tyshynsky, L. Ai, and J. K. Mueller, "Transcranial focused ultrasound neuromodulation of the human primary motor cortex," Scientific reports, vol. 8, no. 1, pp. 1-14, 2018

[55] N. Grossman, M. S. Okun, and E. S. Boyden, "Translating temporal interference brain stimulation to treat neurological and psychiatric conditions," JAMA neurology, vol. 75, no. 11, pp. 1307-1308, 2018.

[56] B. Howell and C. C. McIntyre, "Feasibility of interferential and pulsed transcranial electrical stimulation for neuromodulation at the human scale," Neuromodulation: Technology at the Neural Interface, 2020.

[57] Y. Huang and L. C. Parra, "Can transcranial electric stimulation with multiple electrodes reach deep targets?" Brain stimulation, vol. 12, no. 1, pp. 30-40, 2019.
[58] E. Mirzakhalili, B. Barra, M. Capogrosso, and S. F. Lempka, "Biophysics of temporal interference stimulation," Cell Systems, vol. 11, no. 6, pp. 557-572, 2020

[59] J. S. Sherfey, A. E. Soplata, S. Ardid, E. A. Roberts, D. A. Stanley, B. R. Pittman-Polletta, and N. J. Kopell, "Dynasim: a matlab toolbox for neural modeling and simulation," Frontiers in Neuroinformatics, vol. 12, p. $10,2018$.

[60] H. Markram, M. Toledo-Rodriguez, Y. Wang, A. Gupta, G. Silberberg, and $\mathrm{C}$. Wu, "Interneurons of the neocortical inhibitory system," Nature reviews neuroscience, vol. 5, no. 10, pp. 793-807, 2004.

[61] D. Terman, J. E. Rubin, A. Yew, and C. Wilson, "Activity patterns in a model for the subthalamopallidal network of the basal ganglia," Journal of Neuroscience, vol. 22, no. 7, pp. 2963-2976, 2002.

[62] J. A. Varela, K. Sen, J. Gibson, J. Fost, L. Abbott, and S. B. Nelson, "A quantitative description of short-term plasticity at excitatory synapses in layer 2/3 of rat primary visual cortex," Journal of Neuroscience, vol. 17, no. 20, pp. 7926-7940, 1997.

[63] D. A. Brown, "Muscarinic acetylcholine receptors (machrs) in the nervous system: some functions and mechanisms," Journal of molecular neuroscience, vol. 41 , no. 3, pp. 340-346, 2010.

[64] M. McCarthy, C. Moore-Kochlacs, X. Gu, E. Boyden, X. Han, and N. Kopell, "Striatal origin of the pathologic beta oscillations in parkinson's disease," Proceedings of the National Academy of Sciences, vol. 108, no. 28, pp. 11620-11625, 2011.

[65] J. E. Fleming, E. Dunn, and M. M. Lowery, "Simulation of closed-loop deep brain stimulation control schemes for suppression of pathological beta oscillations in parkinson's disease," Frontiers in Neuroscience, vol. 14, p. 166, 2020.

[66] J. R. Huguenard and D. A. McCormick, "Simulation of the currents involved in rhythmic oscillations in thalamic relay neurons," Journal of neurophysiology, vol. 68, no. 4, pp. 1373-1383, 1992.

[67] T. Otsuka, F. Murakami, and W.-J. Song, "Excitatory postsynaptic potentials trigger a plateau potential in rat subthalamic neurons at hyperpolarized states," Journal of Neurophysiology, vol. 86, no. 4, pp. 1816-1825, 2001.

[68] T. Otsuka, T. Abe, T. Tsukagawa, and W.-J. Song, "Conductance-based model of the voltage-dependent generation of a plateau potential in subthalamic neurons," Journal of neurophysiology, vol. 92, no. 1, pp. 255-264, 2004

[69] A. A. Kühn, F. Kempf, C. Brücke, L. G. Doyle, I. Martinez-Torres, A. Pogosyan, T. Trottenberg, A. Kupsch, G.-H. Schneider, M. I. Hariz et al., "High-frequency stimulation of the subthalamic nucleus suppresses oscillatory $\beta$ activity in patients with parkinson's disease in parallel with improvement in motor performance," Journal of Neuroscience, vol. 28, no. 24, pp. 6165-6173, 2008.

[70] N. Mallet, A. Pogosyan, L. F. Márton, J. P. Bolam, P. Brown, and P. J. Magill, "Parkinsonian beta oscillations in the external globus pallidus and their relationship with subthalamic nucleus activity," Journal of neuroscience, vol. 28, no. 52, pp. 14245-14258, 2008.

[71] N. Mallet, A. Pogosyan, A. Sharott, J. Csicsvari, J. P. Bolam, P. Brown, and P. J. Magill, "Disrupted dopamine transmission and the emergence of exaggerated beta oscillations in subthalamic nucleus and cerebral cortex," Journal of Neuroscience, vol. 28, no. 18, pp. 4795-4806, 2008.

[72] G. C. McConnell, R. Q. So, J. D. Hilliard, P. Lopomo, and W. M. Grill, "Effective deep brain stimulation suppresses low-frequency network oscillations in the basal ganglia by regularizing neural firing patterns," Journal of Neuroscience, vol. 32, no. 45, pp. 15657-15668, 2012.

[73] Y. Tachibana, H. Kita, S. Chiken, M. Takada, and A. Nambu, "Motor cortical control of internal pallidal activity through glutamatergic and gabaergic inputs in awake monkeys," European Journal of Neuroscience, vol. 27, no. 1, pp. 238-253, 2008.

[74] P. P. Mitra and H. Bokil, Observed brain dynamics. Oxford University Press, 2008.

[75] T. Tarnaud, W. Joseph, R. Schoeters, L. Martens, and E. Tanghe, "Membrane charge oscillations during ultrasonic neuromodulation by intramembrane cavitation," IEEE Transactions on Biomedical Engineering, 2020, under review.

[76] _ - "Seconic: Towards multi-compartmental models for ultrasonic brain stimulation by intramembrane cavitation," Journal of Neural Engineering, vol. 17, no. 5, p. 056010, 2020.

[77] M. Vöröslakos, Y. Takeuchi, K. Brinyiczki, T. Zombori, A. Oliva A. Fernández-Ruiz, G. Kozák, Z. T. Kincses, B. Iványi, G. Buzsáki et al., "Direct effects of transcranial electric stimulation on brain circuits in rats and humans," Nature communications, vol. 9, no. 1, pp. 1-17, 2018.

[78] "Food and drug administration, U.S. Department of Health and Human Services marketing clearance of diagnostic 
ultrasound systems and transducers," https://www.fda. gov/regulatory-information/search-fda-guidance-documents/ marketing-clearance-diagnostic-ultrasound-systems-and-transducers accessed: 24 November 2021.

[79] H. Kita and T. Kita, "Cortical stimulation evokes abnormal responses in the dopamine-depleted rat basal ganglia," Journal of Neuroscience, vol. 31, no. 28, pp. 10311-10322, 2011.

[80] E. Moro, R. Esselink, J. Xie, M. Hommel, A. Benabid, and P. Pollak, "The impact on parkinson's disease of electrical parameter settings in stn stimulation," Neurology, vol. 59, no. 5, pp. 706-713, 2002.

[81] V. A. Jourdain, G. Schechtmann, and T. Di Paolo, "Subthalamotomy in the treatment of parkinson's disease: clinical aspects and mechanisms of action: a review," Journal of Neurosurgery, vol. 120, no. 1, pp. 140-151, 2014.

[82] P. J. Hahn and C. C. McIntyre, "Modeling shifts in the rate and pattern of subthalamopallidal network activity during deep brain stimulation," Journal of computational neuroscience, vol. 28, no. 3, pp. 425-441, 2010.

[83] S. Miocinovic, M. Parent, C. R. Butson, P. J. Hahn, G. S. Russo, J. L. Vitek, and C. C. McIntyre, "Computational analysis of subthalamic nucleus and lenticular fasciculus activation during therapeutic deep brain stimulation," Journal of neurophysiology, vol. 96, no. 3, pp. 1569-1580, 2006.

[84] C. C. McIntyre, W. M. Grill, D. L. Sherman, and N. V. Thakor, "Cellular effects of deep brain stimulation: model-based analysis of activation and inhibition," Journal of neurophysiology, vol. 91, no. 4, pp. 1457-1469, 2004.

[85] T. Lemaire, E. Vicari, E. Neufeld, N. Kuster, and S. Micera, "Morphosonic: A morphologically structured intramembrane cavitation model reveals fiber-specific neuromodulation by ultrasound," Iscience, vol. 24 no. 9, p. 103085, 2021.

[86] P. Jin, L. Y. Jan, and Y.-N. Jan, "Mechanosensitive ion channels: Structural features relevant to mechanotransduction mechanisms," Annual Review of Neuroscience, vol. 43, 2020.

[87] A. El Hady and B. B. Machta, "Mechanical surface waves accompany action potential propagation," Nature communications, vol. 6, p. 6697, 2015.

[88] J. Engelbrecht, T. Peets, and K. Tamm, "Electromechanical coupling of waves in nerve fibres," Biomechanics and modeling in mechanobiology, vol. 17, no. 6, pp. 1771-1783, 2018.

[89] M. M. Rvachev, "On axoplasmic pressure waves and their possible role in nerve impulse propagation," Biophysical Reviews and Letters, vol. 5, no. 02, pp. 73-88, 2010.

[90] J. Cao and P. Grover, "Stimulus: Noninvasive dynamic patterns of neurostimulation using spatio-temporal interference," IEEE Transactions on Biomedical Engineering, vol. 67, no. 3, pp. 726-737, 2019.

[91] S. Rampersad, B. Roig-Solvas, M. Yarossi, P. P. Kulkarni, E. Santarnecchi, A. D. Dorval, and D. H. Brooks, "Prospects for transcranial temporal interference stimulation in humans: a computational study," NeuroImage, vol. 202, p. 116124, 2019.

[92] Y. Huang, A. Datta, and L. C. Parra, "Optimization of interferential stimulation of the human brain with electrode arrays," Journal of neural engineering, vol. 17, no. 3, p. 036023, 2020.

[93] X. Zhu, Y. Li, L. Zheng, B. Shao, X. Liu, C. Li, Z.-G. Huang, T. Liu, and J. Wang, "Multi-point temporal interference stimulation by using each electrode to carry different frequency currents," Ieee Access, vol. 7, pp. 168 839-168 848, 2019.

[94] S. Lee, C. Lee, J. Park, and C.-H. Im, "Individually customized transcranial temporal interference stimulation for focused modulation of deep brain structures: a simulation study with different head models," Scientific reports, vol. 10, no. 1, pp. 1-11, 2020.

[95] J. Dmochowski and M. Bikson, "Noninvasive neuromodulation goes deep," Cell, vol. 169, no. 6, pp. 977-978, 2017.

[96] J. Cao and P. Grover, "Do single neuron models exhibit temporal interference stimulation?" in 2018 IEEE Biomedical Circuits and Systems Conference (BioCAS). IEEE, 2018, pp. 1-4.

[97] J. Cao, B. Doiron, C. Goswami, and P. Grover, "The mechanics of temporal interference stimulation," bioRxiv, 2020. 